\title{
Travelling waves in a nonlocal reaction-diffusion equation as a model for a population structured by a space variable and a phenotypical trait
}

\author{
Matthieu Alfaro ${ }^{1}$, Jérôme Coville ${ }^{2}$ and Gaël Raoul ${ }^{3}$.
}

\begin{abstract}
We consider a nonlocal reaction-diffusion equation as a model for a population structured by a space variable and a phenotypical trait. To sustain the possibility of invasion in the case where an underlying principal eigenvalue is negative, we investigate the existence of travelling wave solutions. We identify a minimal speed $c^{*}>0$, and prove the existence of waves when $c \geq c^{*}$ and the non existence when $0 \leq c<c^{*}$.
\end{abstract}

Key Words: structured population, travelling waves, nonlocal reaction-diffusion equation.

AMS Subject Classifications: 35Q92, 45K05, 35C07.

\section{Introduction}

\subsection{Setting of the problem}

In this paper we are interested in propagation phenomena for nonlocal reaction-diffusion equations of the form

$\partial_{t} n(t, x, y)-\Delta_{x, y} n(t, x, y)=\left(r(y-B x \cdot e)-\int_{\mathbb{R}} k\left(y-B x \cdot e, y^{\prime}-B x \cdot e\right) n\left(t, x, y^{\prime}\right) d y^{\prime}\right) n(t, x, y)$,

where $(x, y) \in \mathbb{R}^{d} \times \mathbb{R}, e \in S^{d-1}, B \geq 0, r: \mathbb{R} \rightarrow \mathbb{R}$ and $k: \mathbb{R}^{2} \rightarrow \mathbb{R}^{+}$.

Such equations have appeared in some population dynamic models, see [38], [41], [40], [35]. In this context, $n(t, x, y)$ denotes a density of population structured by a spatial variable $x \in \mathbb{R}^{d}$ and by a phenotypical trait $y \in \mathbb{R}$. The population is then submitted to four essential processes: spatial dispersion, mutations, growth and competition. The spatial dispersion and the mutations are modelled by diffusion operators. The growth rate of the population at a location $x$ and trait $y$ is given - for all times $t$ - by $r(y-B x \cdot e)$, where $r$ is typically negative outside a bounded interval. This corresponds to a population living in an environmental cline: to survive at the location $x$, an individual must have a trait close to $y=B x \cdot e$. Therefore, to be able to invade the environment, the population needs to evolve. Finally, we consider a logistic regulation of the population density that is local in the spatial variable and nonlocal in the trait. In other words, we consider that there exists an intra-specific competition (for e.g. food) at each location, which may

\footnotetext{
${ }^{1}$ I3M, Université de Montpellier 2, CC051, Place Eugène Bataillon, 34095 Montpellier Cedex 5, France. E-mail: malfaro@math.univ-montp2.fr

${ }^{2}$ Equipe BIOSP, INRA Avignon, Domaine Saint Paul, Site Agroparc, 84914 Avignon Cedex 9, France. E-mail: jerome.coville@avignon.inra.fr

${ }^{3}$ Centre d'Écologie Fonctionnelle et Évolutive, UMR 5175, CNRS, 1919 Route de Mende, 34293 Montpellier, France. E-mail: raoul@cefe.cnrs.fr .
} 
depend on the traits of the competitors. For a rigorous derivation of this model from individual based models, we refer to [16]. In Section 2, we will discuss in more details the biological aspects of our work.

The existence of global solutions for the Cauchy problem and of non trivial steady states for (1) have been investigated respectively in [41] and [2]. Also, numerical simulations (see e.g. [38], [41], [40]) show that the population can either go extinct, or propagate while adapting to local environments.

The aim of this work is to analyze this propagation phenomena through the study of travelling front solutions. The travelling front solutions are particular solution of (1) describing the transition at a constant speed $c$ from one stationary solution to another one. Such solutions have proved in numerous situations their utility in describing the dynamics of a population modelled by a reaction diffusion equation. In the case of the classical Fisher-KPP equation

$$
\partial_{t} n-\Delta n=(1-n) n,
$$

we refer among others to [23], [33], [3] [43]: there exists planar fronts $\phi(x . e-c t)$ connecting 0 to 1 , for all speed $c \geq c^{*}=\sqrt{2}$. Moreover, the minimal speed of the front $c^{*}$ corresponds to the so called spreading speed of propagation. Travelling front solutions in heterogeneous versions of (2) with periodicity in space, in time, or more general media are studied in [12], [28], [44], [6], [8], [36], [37]. Nonlocal versions of (2) where the Laplace operator is replaced by a nonlocal operator are studied in [19], [17, 18], [42]. For very general reaction diffusion equations, we refer to [7] for a definition of generalized transition waves and their properties.

It is worth noticing that when the competition term is replaced by a local (in $x$ and $y$ ) density regulation, equation (1) becomes the following heterogeneous reaction diffusion equation

$$
\partial_{t} n(t, x, y)-\Delta_{x, y} n(t, x, y)=(r(y-B x \cdot e)-h(y-B x \cdot e) n(t, x, y)) n(t, x, y),
$$

which was recently investigated by Berestycki and Chapuisat [5]: they prove the existence of a critical speed $c^{*}$, for which there exists a travelling front of $(3)$ for any speed $c \geq c^{*}$.

As far as nonlocal equations of the form (1) are concerned, far less seems to be known in the literature. The travelling wave analysis has been done either using a formal Hamilton-Jacobi approach [15] for a model close to (1), or for a population structured by one variable only, that is $n=n(t, x)$, submitted to a nonlocal competition [9]. To our knowledge, there is no result on the existence of travelling waves for (1) and related models.

\subsection{Assumptions and main results}

As suggested by the numerical simulations mentioned above, we expect that during an invasion, the population adapts locally to the environmental gradient. To observe travelling waves of (1), we therefore perform the change of variable

$$
\tilde{n}(t, x, z)=n(t, x, z+B x \cdot e) .
$$

Then (1) is recast as

$$
\partial_{t} \tilde{n}(t, x, z)-\tilde{\mathcal{E}}(\tilde{n})(t, x, z)=\left(r(z)-\int_{\mathbb{R}} k\left(z, z^{\prime}\right) \tilde{n}\left(t, x, z^{\prime}\right) d z^{\prime}\right) \tilde{n}(t, x, z),
$$

where $\tilde{\mathcal{E}}(\tilde{n}):=\Delta_{x} \tilde{n}+\left(B^{2}+1\right) \tilde{n}_{z z}-2 B \partial_{z}\left(\nabla_{x} \tilde{n} \cdot e\right)$. Since $(1)$ is invariant under any rotation in $\mathbb{R}^{d}$, without loss of generality we can assume that $e=e_{1}$ so that $\tilde{\mathcal{E}}(\tilde{n})=\Delta_{x} \tilde{n}+\left(B^{2}+1\right) \tilde{n}_{z z}-2 B \partial_{x_{1} z} \tilde{n}$. This operator is elliptic, since the associated matrix has only positive eigenvalues. Looking after travelling wave solutions, we search a speed $c$ and a profile $u(x, z)$ such that $\tilde{n}(t, x, z):=u(x$. $\left.e_{1}-c t, z\right)=u\left(x_{1}-c t, z\right)$ solves (5). For convenience, we drop the numerical subscript and write $x$ instead of $x_{1}$. Hence, we are looking after $(c, u(x, z))$ such that

$$
-\mathcal{E}(u)(x, z)-c u_{x}(x, z)=\left(r(z)-\int_{\mathbb{R}} k\left(z, z^{\prime}\right) u\left(x, z^{\prime}\right) d z^{\prime}\right) u(x, z) \quad \text { in } \mathbb{R}^{2},
$$


where

$$
\mathcal{E}(u):=u_{x x}+\left(B^{2}+1\right) u_{z z}-2 B u_{x z} .
$$

Throughout the paper, we make the following assumption.

Assumption 1.1 (Structure of $r$ and $k$ ) Function $r$ is in the Hölder space $C_{\text {loc }}^{0, \theta}(\mathbb{R})$ for some $0<\theta<1$, and there is $\delta>0$ such that

$$
\forall z \in \mathbb{R}, \quad r(z) \leq \frac{1}{\delta}-\delta z^{2} .
$$

Function $k$ is in the Hölder space $C_{\text {loc }}^{0, \theta}\left(\mathbb{R}^{2}\right)$ and there are $k^{-}>0, k^{+}>0$ such that

$$
\forall\left(z, z^{\prime}\right) \in \mathbb{R}^{2}, \quad k^{-} \leq k\left(z, z^{\prime}\right) \leq k^{+} .
$$

Let us next introduce a principal eigenvalue problem that is necessary to enunciate our main result. For more details on principal eigenvalue problems in general domains we refer to [13], [14] and the references therein.

Definition 1.2 (Principal eigenvalue problem) We denote by $\left(\lambda_{\infty}^{0}, \Gamma_{\infty}^{0}\right) \in \mathbb{R} \times C^{\infty}(\mathbb{R})$ the solution of the principal eigenvalue problem

$$
\left\{\begin{array}{l}
-\left(B^{2}+1\right) \Delta_{z} \Gamma_{\infty}^{0}(z)-r(z) \Gamma_{\infty}^{0}(z)=\lambda_{\infty}^{0} \Gamma_{\infty}^{0}(z) \quad \text { for all } z \in \mathbb{R} \\
\Gamma_{\infty}^{0}(z)>0 \quad \text { for all } z \in \mathbb{R}, \quad \Gamma_{\infty}^{0}(0)=1
\end{array}\right.
$$

Observe that in the case where $r(z)=1-A z^{2}, A>0$, we have $\lambda_{\infty}^{0}=\sqrt{A\left(B^{2}+1\right)}-1$ and $\Gamma_{\infty}^{0}(z)=\exp \left(-\sqrt{\frac{A}{B^{2}+1}} \frac{z^{2}}{2}\right)$ is a Gaussian profile.

We first state that as soon as $\lambda_{\infty}^{0}>0$, extinction of the population occurs.

Proposition 1.3 (Extinction) Assume $\lambda_{\infty}^{0}>0$. For any initial population $n^{0}$ such that

$$
\left\|\frac{n^{0}(x, y)}{\Gamma_{\infty}^{0}(y-B x)}\right\|_{L^{\infty}\left(\mathbb{R}^{2}\right)}<\infty
$$

the solution of (1) with initial condition $n^{0}$ goes extinct exponentially fast as $t \rightarrow \infty$ :

$$
\left\|\frac{n(t, x, y)}{\Gamma_{\infty}^{0}(y-B x)}\right\|_{L^{\infty}\left(\mathbb{R}^{2}\right)}=O\left(e^{-\lambda_{\infty}^{0} t}\right)
$$

Next, we state our main result: as soon as $\lambda_{\infty}^{0}<0$, invasion waves exist. Precisely, the following holds.

Theorem 1.4 (Travelling waves) Assume $\lambda_{\infty}^{0}<0$ and define

$$
c^{*}:=2 \sqrt{\frac{-\lambda_{\infty}^{0}}{B^{2}+1}} .
$$

Then the following holds.

(i) For all $c \geq c^{*}$, there exists a positive $u \in C^{2}\left(\mathbb{R}^{2}\right)$ solution of

$$
-\mathcal{E}(u)(x, z)-c u_{x}(x, z)=\left(r(z)-\int_{\mathbb{R}} k\left(z, z^{\prime}\right) u\left(x, z^{\prime}\right) d z^{\prime}\right) u(x, z) \quad \text { in } \mathbb{R}^{2},
$$

with

$$
\nu \mathbf{1}_{\{(x, z) \in(-\infty, 0] \times[-\nu, \nu]\}} \leq u(x, z) \leq C e^{-K z^{2}},
$$


for some $\nu>0, C>0, K>0$, and

$$
\|u(x, \cdot)\|_{\infty} \rightarrow_{x \rightarrow+\infty} 0, \quad \int_{\mathbb{R}} u(x, z) d z \rightarrow_{x \rightarrow+\infty} 0 .
$$

Additionally, when $c>c^{*}$, there exists $\mu<0$ such that

$$
u(x, z) \leq e^{\mu\left(\sqrt{B^{2}+1} x+\frac{B}{\sqrt{B^{2}+1}} z\right)} \Gamma_{\infty}^{0}(z) .
$$

(ii) When $0 \leq c<c^{*}$, there is no positive solution of (10) such that $\lim _{\inf } x_{x \rightarrow+\infty} u(x, 0)=0$ and $u(x, z) \leq \psi(z)$ for some $\psi \in L^{1}(\mathbb{R})$.

\subsection{Comments}

On the extinction case. The proof of Proposition 1.3 is elementary and we now give the proof. The result is a consequence of the parabolic comparison principle satisfied by the local equation

$$
\partial_{t} \phi(t, x, y)-\Delta_{x, y} \phi(t, x, y)=r(y-B x . e) \phi(t, x, y) .
$$

Indeed, one can check that $M e^{-\lambda_{\infty}^{0} t} \Gamma_{\infty}^{0}(y-B x . e)$ and $n(t, x, y)$ are respectively a super- and a sub-solution of (14) with ordered initial data (for $M$ large enough).

On the construction of waves. Let us first comment on a major difficulty in the construction of travelling fronts. When the competition term is replaced by a local (in $x$ and $y$ ) density regulation, many techniques based on the comparison principle — such as some monotone iterative schemes or the sliding method [11] - can be used to get a priori bounds, existence and monotonicity properties of the solution. Since integro-differential equations with a nonlocal competition term do not satisfy the comparison principle, it is unlikely that such techniques apply here.

It turns out that the considered problem here has some similarities with the case of a population structured by a spatial variable only, that is $n=n(t, x)$, submitted to a nonlocal competition as studied in [9] (see also [1]). In this work, the construction of a travelling front is based on a sequence of approximating problems on intervals $\left(-a_{n}, a_{n}\right)$, with $a_{n} \rightarrow \infty$. Due to the lack of comparison principle for the approximated problem, the construction of a solution is based on a topological degree argument, a method introduced initially in [10].

To construct our fronts, we adopt a similar strategy and consider a sequence of problems in growing boxes $\left(-a_{n}, a_{n}\right) \times\left(-b_{n}, b_{n}\right)$, with a normalization at the origin. In order to make this strategy possible, a key point is to establish a priori estimates, independent on the size of the boxes, on the profile $u$, the speed $c$ and, in particular, the tails of $u$ when $z$ is large. Due to the nature of the considered kernels here, an uniform estimate on $u$ is obtained using a local pointwise $L^{p}$ estimate, whereas the uniform control on $c$ is obtained by showing that our problem does not have a solution if the speed $c$ is too large or if $c=0$. Notice that the latter analysis may also be used to simplify the proof in [9].

Let us highlight that, in contrast with [10] and [9], it is far from obvious that the constructed travelling fronts are monotone w.r.t. $x$ for $x>0$ large enough. Therefore, we shall need an extra work to catch the behavior (12) as $x \rightarrow+\infty$.

Notice also that the comprehension of the behavior of the wave as $x \rightarrow-\infty$ is quite involved. In the related case of the nonlocal Fisher-KPP equation, the positive steady state $u \equiv 1$ may present, for some kernels, a Turing instability (see e.g. [24], [9], [1]). Such a situation may also occur in our context.

Let us also mention that, although we construct fronts without relying on any monotonic properties of the profiles, it is suspected, as in the case of the nonlocal Fisher-KPP equation [9], that there exist monotone and non monotone travelling fronts. The understanding of such issues is quite challenging.

Organization of the paper. In Section 2 we briefly describe the biological context of (1) and give an interpretation of our results. Then, we prove Theorem 1.4 in Sections 3-5. In Section 3, 
we start by deriving some a priori bounds and then, using a Leray-Schauder topological degree argument, we construct a solution in a bounded box. In Section 4, we let the box tend to $\mathbb{R}^{2}$ and obtain a wave, which turns out to be the one with minimal speed $c=c^{*}$. We also show the non existence of waves with speed $0 \leq c<c^{*}$. Lastly, we construct faster waves $c>c^{*}$ in Section 5 .

\section{Biological interpretation of the results}

In this section we briefly precise the biological context of (1).

In the present paper, we are interested in biological invasions involving darwinian evolution. Species invading new territories often face environmental gradients of e.g. temperature, luminosity, antibiotic chemicals. Experimentally, it is well documented that invasive species then evolve during their range expansion [22], [30], to adapt to local conditions. To understand the speed, or even the success of an invasion, one should thus consider the dispersion, birth and death processes, but should also take into account evolution [26], [31], [27]. Those questions become especially important in the context of the global warming [20], [21]: the favorable environmental conditions of many species move towards the north, implying important changes in species' range. It is also of great importance for the evolution of resistance of bacteria to antibiotics [27].

More generally, many evolutionary biology questions involve spatially structured populations, while most existing models either neglect the spacial structure of the population, or largely simplify it. New theoretical tools are then needed, and structured population models are natural candidates: they enable the modelling of all the biological phenomena mentioned above, and numerical simulations show that they are able to reproduce interesting features. Analyzing this type of model is however challenging, even in a homogeneous setting, see e.g. [29], [34]. This work, as well as the results of [15] are first steps in the mathematical understanding of the dynamics of those models.

The main application of our result concerns asexual populations living in an environmental cline. The simplest model then writes

$$
\begin{aligned}
\partial_{t} n(t, x, y) \quad & -\frac{\sigma_{x}^{2}}{2} \Delta_{x} n(t, x, y)-\frac{\sigma_{m}^{2}}{2} \Delta_{y} n(t, x, y)= \\
& \left(r_{\max }-\frac{1}{2 V_{s}}(y-b x)^{2}-\frac{1}{K} \int_{\mathbb{R}} n\left(t, x, y^{\prime}\right) d y^{\prime}\right) n(t, x, y),
\end{aligned}
$$

where $\sigma_{x}, \sigma_{m}$ describe respectively the diffusion rate and the mutation rate of the population, $\frac{1}{2 V_{s}}$ is the strength of the selection, $b$ is the steepness of the environment cline, and $K$ the carrying capacity of the environment. After the rescaling $n(t, x, y)=\tilde{n}\left(r_{\max } t, \frac{\sqrt{2 r_{\max }}}{\sigma_{x}} x, \frac{\sqrt{2 r_{\max }}}{\sigma_{m}} y\right)$, we see that $\tilde{n}$ solves (1) with

$$
r(y)=1-A y^{2}, \quad A=\frac{\sigma_{m}^{2}}{4 r_{\max }^{2} V_{s}}, \quad B=\frac{\sigma_{x}}{\sigma_{m}} b, \quad k \equiv \frac{1}{K r_{\max }} .
$$

The population then gets extinct if $A\left(B^{2}+1\right)>1$, while if $A\left(B^{2}+1\right)<1$, invasion fronts exist, with a minimal propagation speed (in the original variables)

$$
\sqrt{2 r_{\max }} \sigma_{x}\left(1-\frac{\sigma_{m}}{2 r_{\max } \sqrt{V_{s}}} \sqrt{\left(b \frac{\sigma_{x}}{\sigma_{m}}\right)^{2}+1}\right)^{1 / 2}\left(\left(b \frac{\sigma_{x}}{\sigma_{m}}\right)^{2}+1\right)^{-1 / 2} .
$$

Remark 2.1 There exists thus only two dynamics: either the population gets extinct, or it succeeds to invade the whole territory. The situation of asexual populations is then very different from the case of sexual populations (see [32], [35]), where populations surviving with a limited range only are possible.

Notice also that during invasions, the dispersion of individuals can evolve (see e.g. [39]). Our result does not apply to this problem, and we refer to [4], [15] for such a situation. 


\section{The problem in a bounded box}

\subsection{On some principal eigenvalue problems}

We first introduce some principal eigenvalue problems, whose eigenfunctions will serve as boundary conditions when stating the travelling wave problem in a bounded box.

For $\nu \in[0, \delta)$, where $\delta>0$ is as in Assumption 1.1, we denote by $\left(\lambda_{\infty}^{\nu}, \Gamma_{\infty}^{\nu}\right)$ the solution of the principal eigenvalue problem

$$
\left\{\begin{array}{l}
-\left(B^{2}+1\right) \Delta_{z} \Gamma_{\infty}^{\nu}(z)-\left(r(z)+\nu z^{2}\right) \Gamma_{\infty}^{\nu}(z)=\lambda_{\infty}^{\nu} \Gamma_{\infty}^{\nu}(z) \quad \text { for all } z \in \mathbb{R} \\
\Gamma_{\infty}^{\nu}(z)>0 \quad \text { for all } z \in \mathbb{R}, \quad \Gamma_{\infty}^{\nu}(0)=1 .
\end{array}\right.
$$

Notice that this definition is coherent with (8), and for any $\nu \in[0, \delta)$, we have $\lambda_{\infty}^{\nu} \leq \lambda_{\infty}^{0}<0$. Also, for $\nu \in[0, \delta)$ and $b>0$, we define $\left(\lambda_{b}^{\nu}, \Gamma_{b}^{\nu}\right)$ as the solution of the principal eigenvalue problem

$$
\left\{\begin{array}{l}
-\left(B^{2}+1\right) \Delta_{z} \Gamma_{b}^{\nu}(z)-\left(r(z)+\nu z^{2}\right) \Gamma_{b}^{\nu}(z)=\lambda_{b}^{\nu} \Gamma_{b}^{\nu}(z) \quad \text { for all } z \in(-b, b) \\
\Gamma_{b}^{\nu}( \pm b)=0 \\
\Gamma_{b}^{\nu}(z)>0 \quad \text { for all } z \in(-b, b), \quad \Gamma_{b}^{\nu}(0)=1
\end{array}\right.
$$

Let us observe that $b<b^{\prime}$ implies $\lambda_{\infty}^{\nu}<\lambda_{b^{\prime}}^{\nu}<\lambda_{b}^{\nu}$, and that $\lambda_{b}^{\nu} \rightarrow \lambda_{\infty}^{\nu}$ as $b \rightarrow \infty$. To construct the travelling waves, we will use the eigenfunctions $\Gamma_{b}^{\delta / 3}$, for $b>0$ as a boundary value. To bound from above those functions independently of $b>0$, we will also use the functions $\Gamma_{\infty}^{2 \delta / 3}$. Notice that $-\max _{z \in \mathbb{R}} r(z) \leq \lambda_{\infty}^{2 \delta / 3}<\lambda_{\infty}^{\delta / 3}<0$. To show that $\Gamma_{\infty}^{2 \delta / 3}$ is integrable, we define $C:=\max _{[-\bar{z}, \bar{z}]} \Gamma_{\infty}^{2 \delta / 3}$, where $\bar{z}:=\frac{\sqrt{6}}{\delta}$, and $\psi(z):=C \exp \left(-\sqrt{\frac{\delta}{B^{2}+1}} \frac{z^{2}-\bar{z}^{2}}{2 \sqrt{6}}\right)$, so that $\psi( \pm \bar{z})=C$ and

$$
-\left(B^{2}+1\right) \Delta_{z} \psi(z)-\left(r(z)+\frac{2 \delta}{3} z^{2}\right) \psi(z) \geq\left(\frac{\delta}{6} z^{2}-\frac{1}{\delta}\right) \psi(z) \geq 0,
$$

for all $z \in(-\infty,-\bar{z}) \cup(\bar{z}, \infty)$. Since in $(-\infty,-\bar{z}) \cup(\bar{z}, \infty)$ we have $r(z)+\frac{2 \delta}{3} z^{2} \leq \frac{1}{\delta}-\frac{\delta}{3} z^{2} \leq 0$, the comparison principle then applies to $(15)$ on $(-\infty,-\bar{z}) \cup(\bar{z}, \infty)$, and yields $\Gamma_{\infty}^{2 \delta / 3}(z) \leq \psi(z)$ for all $z \in(-\infty,-\bar{z}) \cup(\bar{z}, \infty)$. As a result, for some constant which we denote again by $C$, we have

$$
\Gamma_{\infty}^{2 \delta / 3}(z) \leq C \exp \left(-\sqrt{\frac{\delta}{B^{2}+1}} \frac{z^{2}}{2 \sqrt{6}}\right) \quad \text { for all } z \in \mathbb{R}
$$

which implies in turn that $\Gamma_{\infty}^{2 \delta / 3} \in L^{1}(\mathbb{R})$.

For a given $b_{0}>0$, we now use a similar argument to control the functions $\Gamma_{b}^{\delta / 3}(z)$ uniformly w.r.t. $b \in\left[b_{0}, \infty\right]$. When $z$ lies in $[-\bar{z}, \bar{z}]$, where $\bar{z}:=\max \left\{\sqrt{\frac{3\left(\lambda_{b_{0}}^{\delta / 3}-\lambda_{\infty}^{2 \delta / 3}\right)}{\delta}}, \sqrt{\frac{3}{2 \delta}\left(\lambda_{b_{0}}^{\delta / 3}+\frac{1}{\delta}\right)}\right\}$, the coefficients of the equations in (15) and (16) are uniformly bounded w.r.t. $b \in\left[b_{0}, \infty\right]$. Therefore the Harnack inequality implies that there is $C>0$ such that $\Gamma_{b}^{\delta / 3}(z) \leq C$, for all $z \in[-\bar{z}, \bar{z}]$, all $b \in\left[b_{0}, \infty\right]$. By the definition of $\bar{z}$ we see that, on the one hand, $\Gamma_{\infty}^{2 \delta / 3}$ is a super-solution for (15) and $(16)$ - with $\nu=\frac{\delta}{3}$ - on $(-\infty,-\bar{z}) \cup(\bar{z}, \infty)$ and that, on the other hand, the comparison principle applies. Therefore, there exists $\bar{C}>0$ such that

$$
\Gamma_{b}^{\delta / 3}(z) \leq \bar{C} \Gamma_{\infty}^{2 \delta / 3}(z) \leq \bar{C}\left\|\Gamma_{\infty}^{2 \delta / 3}\right\|_{\infty} \quad \text { for all } z \in \mathbb{R}, b \in\left[b_{0}, \infty\right]
$$

In particular, we have

$$
\int_{\mathbb{R}} \Gamma_{b}^{\delta / 3}(z) d z \leq m_{\Gamma}:=\bar{C} \int_{\mathbb{R}} \Gamma_{\infty}^{2 \delta / 3}(z) d z<\infty \quad \text { for all } b \in\left[b_{0}, \infty\right]
$$




\subsection{The problem in a box}

For $a>0, b>0$ and $\varepsilon \in(0,1)$, we consider the problem of finding a speed $c \in \mathbb{R}$ and a real function $u(x, z)$, defined for $(x, z) \in[-a, a] \times[-b, b]$, such that

$$
P(a, b, \varepsilon) \begin{cases}-\mathcal{E}(u)(x, z)-c u_{x}(x, z) & \\ =\mathbf{1}_{\{u(x, z) \geq 0\}}\left(r(z)-\int_{-b}^{b} k\left(z, z^{\prime}\right) u\left(x, z^{\prime}\right) d z^{\prime}\right) u(x, z) & \text { in } Q \\ u(x, z)=\mathbf{1}_{\{x=-a\}}(x) \Gamma_{b}^{\delta / 3}(z) & \text { on } \partial Q \\ u(0,0)=\varepsilon, & \end{cases}
$$

where $Q:=(-a, a) \times(-b, b)$. The elliptic operator is given by

$$
-\mathcal{E}(u)=-u_{x x}-\left(B^{2}+1\right) u_{z z}+2 B u_{x z} .
$$

If $(c, u)$ is a solution achieving a negative minimum at $\left(x_{m}, z_{m}\right)$ then, from the boundary conditions we deduce that $\left(x_{m}, z_{m}\right)$ lies in the interior of the rectangle, and that $-\mathcal{E}(u)-c u_{x}=0$ on a neighborhood of $\left(x_{m}, z_{m}\right)$. The maximum principle thus implies $u \equiv u\left(x_{m}, z_{m}\right)$, which cannot be. Therefore any solution of $P(a, b, \varepsilon)$ satisfies $u \geq 0$ and, by the strong maximum principle,

$$
u>0 \quad \text { and } \quad-\mathcal{E}(u)(x, z)-c u_{x}(x, z)=\left(r(z)-\int_{-b}^{b} k\left(z, z^{\prime}\right) u\left(x, z^{\prime}\right) d z^{\prime}\right) u(x, z) \quad \text { in } Q .
$$

In the following of the section, we shall construct a solution to $P(a, b, \varepsilon)$ via a Leray-Schauder topological degree argument. To make this possible, we consider a family of problems as follows. For $a>0, b>0$ and $\tau \in[0,1]$, we consider the problem of finding a speed $c \in \mathbb{R}$ and a nonnegative real function $u(x, z)$ such that

$$
P_{\tau}(a, b) \begin{cases}-\mathcal{E}(u)(x, z)-c u_{x}(x, z) & \text { in } Q \\ =\left(r(z)-\tau \int_{-b}^{b} k\left(z, z^{\prime}\right) u\left(x, z^{\prime}\right) d z^{\prime}-\gamma(1-\tau) u(x, z)\right) u(x, z) & \text { on } \partial Q \\ u(x, z)=\mathbf{1}_{\{x=-a\}}(x) \Gamma_{b}^{\delta / 3}(z)\end{cases}
$$

where $\gamma>0$ will be specified later (see Lemma 3.7). Note that $P_{0}(a, b)$ reduces to a local problem, and that solving $P(a, b, \varepsilon)$ is equivalent to solving $P_{1}(a, b)$ with the additional normalization condition $u(0,0)=\varepsilon$.

Remark 3.1 A first natural idea to define a family of problems would be to consider $-\mathcal{E}(u)-c u_{x}=$ $\tau\left(r(z)-\int_{-b}^{b} k\left(z, z^{\prime}\right) u\left(x, z^{\prime}\right) d z^{\prime}\right) u$. But then we cannot get a uniform w.r.t. $0 \leq \tau \leq 1$ control of the tails of $u$ (see Lemma 3.4), which is crucial to derive e.g. a lower bound on the standing waves (see Lemma 3.6). This is the reason why we consider the family $P_{\tau}(a, b)$ as above. Therefore, the topological degree argument (see subsection 3.6) is rather involved and requires to analyze a whole family of local problems (see Lemma 3.8).

\subsection{A priori estimates for $u$}

We provide a priori bounds for the profile $u$ of solutions to $P_{\tau}(a, b)$. When $0 \leq \tau \leq 1 / 2$, the local part of the equation shall be enough to derive Lemma 3.3. On the other hand, when $1 / 2 \leq \tau \leq 1$, the nonlocal part is quite relevant and we first need to control the vertical mass of $u$, namely

$$
m(x):=\int_{-b}^{b} u(x, z) d z
$$


Lemma 3.2 (A priori bound for the mass) For all $a>0, b \geq b_{0}>0,1 / 2 \leq \tau \leq 1$, any solution $(c, u)$ of $P_{\tau}(a, b)$ satisfies

$$
0 \leq \int_{-b}^{b} u(x, z) d z \leq \max \left(\frac{2 \max _{\mathbb{R}} r}{k^{-}}, m_{\Gamma}\right), \quad \forall x \in[-a, a] .
$$

Proof. Integrating w.r.t. $z$ the inequality $-u_{x x}-c u_{x} \leq\left(\max _{\mathbb{R}} r-\frac{1}{2} k^{-} m(x)\right) u+\left(B^{2}+1\right) u_{z z}-$ $2 B u_{x z}$, we get

$$
\begin{aligned}
-m^{\prime \prime}(x)-c m^{\prime}(x) \leq & \left(\max _{\mathbb{R}} r-\frac{1}{2} k^{-} m(x)\right) m(x)+\left(B^{2}+1\right)\left(u_{z}(x, b)-u_{z}(x,-b)\right) \\
& -2 B\left(u_{x}(x, b)-u_{x}(x,-b)\right) .
\end{aligned}
$$

Since $u_{z}(x, b) \leq 0, u_{z}(x,-b) \geq 0$ and $u_{x}(x, b)=u_{x}(x,-b)=0$, the mass satisfies the Fisher-KPP inequality $-m^{\prime \prime}-c m^{\prime} \leq\left(\max _{\mathbb{R}} r-\frac{1}{2} k^{-} m\right) m$. Since $m(-a) \leq m_{\Gamma}$ (see (19)) and $m(a)=0$, the maximum principle concludes the proof of the lemma.

The above nonlocal control now provides the following a priori bound for $u$.

Lemma 3.3 (A priori bound for $u$ ) There exists $M>0$ such that, for all $a>0, b \geq b_{0}>0$, $0 \leq \tau \leq 1$, any solution $(c, u)$ of $P_{\tau}(a, b)$ with $0 \leq c \leq c^{*}+1$ satisfies

$$
0 \leq u(x, z) \leq M, \quad \forall(x, z) \in[-a, a] \times[-b, b] .
$$

Proof. For $0 \leq \tau \leq 1 / 2$, one keeps the local part and writes $-\mathcal{E}(u)-c u_{x} \leq\left(\max _{\mathbb{R}} r-\frac{\gamma}{2} u\right) u$; recalling (18), the maximum principle then gives a control of $u$ by $\max \left(\frac{2 \max r}{\gamma}, \bar{C}\left\|\Gamma_{\infty}^{2 \delta / 3}\right\|_{\infty}^{2}\right)$.

Next, for $1 / 2 \leq \tau \leq 1$, let us denote by $\left(x_{M}, z_{M}\right)$ a point where $u$ achieves its maximum $M$, and by $B_{r}$ the ball centered at $\left(x_{M}, z_{M}\right)$ with radius $r>0$. Note that $0 \leq u \leq \bar{C}\left\|\Gamma_{\infty}^{2 \delta / 3}\right\|_{\infty}$ on $\partial Q$. The function $w:=u-\bar{C}\left\|\Gamma_{\infty}^{2 \delta / 3}\right\|_{\infty}$ therefore satisfies

$$
\begin{cases}-\mathcal{E}(w)-c w_{x}-\left(\max _{\mathbb{R}} r\right) w \leq C_{0}:=\left(\max _{\mathbb{R}} r\right) \bar{C}\left\|\Gamma_{\infty}^{2 \delta / 3}\right\|_{\infty} & \text { in } Q \\ w \leq 0 & \text { on } \partial Q .\end{cases}
$$

From the local maximum principle [25, Theorem 9.20] and it extension up to balls intersecting the boundary of the domain [25, Theorem 9.26], we infer that

$$
\sup _{B_{1 / 2} \cap Q} w \leq C_{1}\left(\frac{1}{\left|B_{1}\right|} \int_{B_{1} \cap Q} w^{+}+C_{2}\left\|C_{0}\right\|_{L^{N}\left(B_{1} \cap Q\right)}\right),
$$

where $C_{1}=C_{1}(B)$ and $C_{2}=C_{2}(B)$ are positive constants. Notice that $C_{1}$ does not depend on $c$, which is a coefficient of the operator $L(w):=\mathcal{E}(w)+c w_{x}+w$, because $c$ belongs to a bounded interval, namely $\left[0, c^{*}+1\right]$. Using successively $u \geq 0$ and Lemma 3.2 we deduce that

$$
\int_{B_{1} \cap Q} w^{+} \leq \int_{B_{1} \cap Q} u+\int_{B_{1} \cap Q} \bar{C}\left\|\Gamma_{\infty}^{2 \delta / 3}\right\|_{\infty} \leq 2 \max \left(\frac{2 \max _{\mathbb{R}} r}{k^{-}}, m_{\Gamma}\right)+\bar{C}\left\|\Gamma_{\infty}^{2 \delta / 3}\right\|_{\infty}\left|B_{1}\right| .
$$

Recalling that $M=\max u$ is achieved at the center of the ball $B_{1 / 2}$, we deduce form the upper estimates that

$$
M \leq \bar{C}\left\|\Gamma_{\infty}^{2 \delta / 3}\right\|_{\infty}+C_{1}\left(\frac{2}{\left|B_{1}\right|} \max \left(\frac{2 \max _{\mathbb{R}} r}{k^{-}}, m_{\Gamma}\right)+\bar{C}\left\|\Gamma_{\infty}^{2 \delta / 3}\right\|_{\infty}+C_{2} C_{0}\left|B_{1}\right|\right) .
$$

This concludes the proof of the lemma.

We now provide a control of the tails of the solutions as $|z| \rightarrow \infty$ by appropriate Gaussian functions (recall estimate (17)). 
Lemma 3.4 (Gaussian control of the tails of $u$ ) There exists $\bar{M}>0$ such that, for all $a>0$, $b \geq b_{0}>0,0 \leq \tau \leq 1$, any solution $(c, u)$ of $P_{\tau}(a, b)$ with $0 \leq c \leq c^{*}+1$ satisfies

$$
0 \leq u(x, z) \leq \bar{M} \Gamma_{\infty}^{2 \delta / 3}(z), \quad \forall(x, z) \in[-a, a] \times[-b, b] .
$$

Proof. First observe that

$$
-\mathcal{E}(u)-c u_{x}-r(z) u \leq 0 \quad \text { on } Q, \quad u \leq \bar{C} \Gamma_{\infty}^{2 \delta / 3} \quad \text { on } \partial Q .
$$

Define $\bar{\phi}(x, z)=\bar{\phi}(z):=\bar{M} \Gamma_{\infty}^{2 \delta / 3}(z)$, with $\bar{M}>0$ to be specified later. Recall that $\Gamma_{\infty}^{2 \delta / 3}$ solves (15) so that $-\mathcal{E}(\bar{\phi})-c \bar{\phi}_{x}-r(z) \bar{\phi}=\left(\frac{2 \delta}{3} z^{2}+\lambda_{\infty}^{2 \delta / 3}\right) \bar{\phi}$. Therefore, if $\beta:=\max \left(\frac{1}{\delta}, \sqrt{\frac{-3 \lambda_{\infty}^{2 \delta / 3}}{2 \delta}}\right)$, we have

$$
-\mathcal{E}(\bar{\phi})-c \bar{\phi}_{x}-r(z) \bar{\phi} \geq 0 \quad \text { on }(-a, a) \times(\beta, b) .
$$

Let us now select

$$
\bar{M}:=\max \left(\bar{C}, \frac{M}{\min _{[-\beta, \beta]} \Gamma_{\infty}^{2 \delta / 3}}\right),
$$

where $M$ is as in the previous lemma. The choice (24) enforces $\bar{\phi}(x, z) \geq \bar{C} \Gamma_{\infty}^{2 \delta / 3}(z) \geq u(x, z)$ on $\{\mp a\} \times[\beta, b] \cup[-a, a] \times\{b\}$, and $\bar{\phi}(x, z) \geq M \geq u(x, z)$ on $[-a, a] \times\{\beta\}$. Hence the comparison principle - note that $r(z) \leq 0$, when $z \geq \beta$ - yields $u \leq \bar{\phi}$ on $[-a, a] \times[\beta, b]$ and, by the choice $(24)$, on $[-a, a] \times[0, b]$. Similarly, $u \leq \bar{\phi}$ on $[-a, a] \times[-b, 0]$. The lemma is proved.

\subsection{A priori estimates for $c$}

We provide a priori bounds for the speed $c$ of solutions to $P_{\tau}(a, b)$. We first show that, roughly speaking, too rapid waves solutions of $P_{\tau}(a, b)$ have too small value at $(x, z)=(0,0)$. We recall that the speed $c^{*}$ was defined in $(9)$.

Lemma 3.5 (A priori upper bound for $c$ ) Let $b>0$ and $\varepsilon \in(0,1)$ be arbitrary. Then there exists $a_{0}=a_{0}(\varepsilon, b)>0$ such that, for all $a \geq a_{0}$, all $0 \leq \tau \leq 1$, any solution $(c, u)$ of $P_{\tau}(a, b)$ with $c>c^{*}$ satisfies $u(0,0)<\varepsilon$ - and therefore cannot solve $P(a, b, \varepsilon)$.

Proof. Let $b>0$ be given. Assume $c>c^{*}$ and let us show that $u(0,0) \rightarrow 0$ as $a \rightarrow \infty$.

The function $u$ satisfies $-\mathcal{E}(u)-c u_{x}-r(z) u \leq 0$ in $Q$. Therefore, changing variables, the function

$$
v(x, y):=u\left(\frac{x-B y}{\sqrt{B^{2}+1}}, \sqrt{B^{2}+1} y\right),
$$

satisfies

$$
-v_{x x}-v_{y y}-c \sqrt{B^{2}+1} v_{x}-r\left(\sqrt{B^{2}+1} y\right) v \leq 0,
$$

in $Q_{1}:=\left\{(x, y):|y|<\frac{b}{\sqrt{B^{2}+1}},\left|\frac{x-B y}{\sqrt{B^{2}+1}}\right|<a\right\}$.

We shall now construct a positive solution. Since $c>c^{*}$, one can select $\mu<0$ such that $\mu^{2}+c \sqrt{B^{2}+1} \mu+\frac{c^{* 2}}{4}\left(B^{2}+1\right)=0$ and define $\varphi(s):=e^{\mu s}$ which solves

$$
-\varphi^{\prime \prime}-c \sqrt{B^{2}+1} \varphi^{\prime}-\frac{c^{* 2}}{4}\left(B^{2}+1\right) \varphi=0 .
$$

Now, let us define

$$
w(x, y):=\kappa_{a} \varphi(x) \Gamma_{\infty}^{0}\left(\sqrt{B^{2}+1} y\right), \quad \kappa_{a}:=\frac{\left\|\Gamma_{b}^{\delta / 3}\right\|_{\infty}}{\min _{[-b, b]} \Gamma_{\infty}^{0}} e^{\mu\left(a \sqrt{B^{2}+1}-\frac{B b}{\sqrt{B^{2}+1}}\right)},
$$


with $\Gamma_{\infty}^{0}$ the eigenfunction appearing in Definition 1.2. Using direct computations and the definition of $c^{*}$ in (9) we see that

$$
-w_{x x}-w_{y y}-c \sqrt{B^{2}+1} w_{x}-r\left(\sqrt{B^{2}+1} y\right) w=0 \quad \text { in } Q_{1} .
$$

We now compare the values of $v$ and $w$ on the boundary. If $(x, y) \in \partial Q_{1}$ is such that $\frac{x-B y}{\sqrt{B^{2}+1}} \neq$ $-a$, then $v(x, y)=0<w(x, y)$. If $(x, y) \in \partial Q_{1}$ is such that $\frac{x-B y}{\sqrt{B^{2}+1}}=-a$ then

$$
v(x, y)=\Gamma_{b}^{\delta / 3}\left(\sqrt{B^{2}+1} y\right) \leq \kappa_{a} e^{\mu\left(-a \sqrt{B^{2}+1}+\frac{B b}{\sqrt{B^{2}+1}}\right)} \min _{[-b, b]} \Gamma_{\infty}^{0} \leq w(x, y) .
$$

As a result, we have $v \leq w$ on $\partial Q_{1}$.

Now a classical argument will imply $v \leq w$ on the whole of $\overline{Q_{1}}$. Indeed, since $v$ is bounded and $w>0$ on $\overline{Q_{1}}$, we can define $\alpha_{0}:=\max \left\{\alpha>0: \alpha v \leq w\right.$ in $\left.\overline{Q_{1}}\right\}>0$. Then $\alpha_{0} v \leq w$ and there is a point $\left(x_{0}, y_{0}\right)$ such that $\alpha_{0} v\left(x_{0}, y_{0}\right)=w\left(x_{0}, y_{0}\right)$. In view of $(26),(27)$ and the strong maximum principle the point $\left(x_{0}, y_{0}\right)$ has to lie on $\partial Q_{1}$, which enforces $\alpha_{0} \geq 1$. Thus $v \leq w$ in $\overline{Q_{1}}$, and

$$
u(0,0)=v(0,0) \leq w(0,0)=\kappa_{a} \rightarrow 0 \text { as } a \rightarrow \infty,
$$

which concludes the proof of the lemma.

Next, we show that standing waves (i.e. $c=0)$ have too large value at $(x, z)=(0,0)$.

Lemma 3.6 (Standing waves: a priori lower bound for $u(0,0)$ ) There is $\varepsilon^{*}>0$ such that if $a, b$ are large enough, then, for all $0 \leq \tau \leq 1$, any standing solution $(c=0, u)$ of $P_{\tau}(a, b)$ satisfies $u(0,0)>\varepsilon^{*}$ - and therefore cannot solve $P(a, b, \varepsilon)$ for any $\varepsilon \in\left(0, \varepsilon^{*}\right)$.

Proof. For $R>0$, let us introduce $\left(\mu_{R}, \Upsilon_{R}\right)$ as the solution of the principal eigenvalue problem

$$
\left\{\begin{array}{l}
-\mathcal{E}\left(\Upsilon_{R}\right)(x, z)-r(z) \Upsilon_{R}(x, z)=\mu_{R} \Upsilon_{R}(x, z) \quad \text { for all }(x, z) \in(-R, R)^{2} \\
\Upsilon_{R}=0 \text { on } \partial\left((-R, R)^{2}\right) \\
\Upsilon_{R}(x, z)>0 \quad \text { for all }(x, z) \in(-R, R)^{2}, \quad \Upsilon_{R}(0,0)=1 .
\end{array}\right.
$$

If $R=\infty$, the above problem is equivalent to (8), and $\mu_{R} \rightarrow \lambda_{\infty}^{0}$ as $R \rightarrow \infty$. Let us therefore fix $R>0$ large enough so that

$$
\lambda_{\infty}^{0} \leq \mu_{R}<\frac{\lambda_{\infty}^{0}}{2}<0
$$

and

$$
k^{+} \int_{[-R, R]^{c}} \bar{M} \Gamma_{\infty}^{2 \delta / 3}(z) d z \leq \frac{-\lambda_{\infty}^{0}}{4} .
$$

Next, let $a \geq R+1, b \geq R+1,0 \leq \tau \leq 1$ be given, and $(c=0, u)$ be a solution of $P_{\tau}(a, b)$. Thanks to the Harnack inequality, there exists $C>0$ (independent of $a$ and $b$ ), such that $\|u\|_{L^{\infty}\left([-R, R]^{2}\right)} \leq C u(0,0)$, and then

$$
\max _{(x, z) \in[-R, R]^{2}}\left(\tau \int_{-b}^{b} k\left(z, z^{\prime}\right) u\left(x, z^{\prime}\right) d z^{\prime}+\gamma(1-\tau) u\right) \leq C\left(2 R k^{+}+\gamma\right) u(0,0)+\frac{-\lambda_{\infty}^{0}}{4},
$$

where we have used Lemma 3.4 and (30). As a result, $u$ satisfies

$$
-\mathcal{E}(u)-r(z) u \geq\left(-C\left(2 R k^{+}+\gamma\right) u(0,0)+\frac{\lambda_{\infty}^{0}}{4}\right) u \quad \text { in }(-R, R)^{2},
$$

and $u>0$ on $\partial\left((-R, R)^{2}\right)$. Hence, if

$$
u(0,0) \leq \frac{-\lambda_{\infty}^{0} / 4}{C\left(2 R k^{+}+\gamma\right)}=: \varepsilon^{*},
$$


$u$ becomes a super-solution for (28) - thanks to (29). We conclude as in the proof of Lemma 3.5: defining $\alpha_{0}:=\max \left\{\alpha>0: \alpha \Upsilon_{R} \leq u\right.$ in $\left.[-R, R]^{2}\right\}>0$ and using the strong maximum principle we see that $\alpha_{0} \Upsilon_{R} \equiv u$ on $[-R, R]^{2}$, which contradicts $u>0$. It follows that $u(0,0)>\varepsilon^{*}$. The lemma is proved.

\subsection{On some related local problems}

First, we show the well-posedness of the local problem $P_{0}(a, b)$.

Lemma 3.7 (Well-posedness for $\tau=0$ ) There exists $\gamma>0$ such that, if $a$, $b$ are large enough and $\varepsilon \in\left(0, \varepsilon^{*}\right)$, there exists a unique $(c, u \geq 0) \in \mathbb{R} \times W^{2, \infty}(Q)$, solution of $P_{0}(a, b)$, namely

$$
P_{0}(a, b) \begin{cases}-\mathcal{E}(u)(x, z)-c u_{x}(x, z)=(r(z)-\gamma u(x, z)) u(x, z) & \text { in } Q \\ u(x, z)=\mathbf{1}_{\{x=-a\}}(x) \Gamma_{b}^{\delta / 3}(z) & \text { on } \partial Q\end{cases}
$$

such that $u(0,0)=\varepsilon$. Moreover, by the above a priori estimates, $0<c \leq c^{*}$ and $0 \leq u \leq M$.

Proof. A standard argument proves that there is a unique positive solution to $P_{0}(a, b)$. For the convenience of the reader let us prove this fact. Since 0 and a large enough positive constant are respectively a sub- and a super-solution of $P_{0}(a, b)$, the existence of a positive solution to $P_{0}(a, b)$ can be obtained using a classical monotone iterative scheme. Also, by the maximum principle, any positive solution of $P_{0}(a, b)$ is bounded. Now let $u$ and $v$ be two bounded positive solutions of $P_{0}(a, b)$. Thanks to the boundary condition and the Hopf lemma the following quantity is well defined:

$$
\tau^{*}:=\inf \{\tau>0: \forall(x, z) \in \bar{Q}, u(x, z) \leq \tau v(x, z)\} .
$$

Assume by contradiction that $\tau^{*}>1$. From the definition of $\tau^{*}$, the boundary condition and the Hopf lemma, there exists $\left(x_{0}, z_{0}\right) \in Q$ such that $u\left(x_{0}, z_{0}\right)=\tau^{*} v\left(x_{0}, z_{0}\right)$. At this point, we get the contradiction

$$
0 \leq \mathcal{E}\left(\tau^{*} v-u\right)\left(x_{0}, z_{0}\right)+c\left(\tau^{*} v-u\right)_{x}\left(x_{0}, z_{0}\right) \leq \gamma \tau^{*}\left(1-\tau^{*}\right) v^{2}\left(x_{0}, z_{0}\right)<0 .
$$

Thus $\tau^{*} \leq 1$ and we have $u \leq v$. By interchanging the role of $u$ and $v$, we get $u \equiv v$. Hence there is a unique positive solution to $P_{0}(a, b)$.

In order to apply a sliding method, we slightly modify the Dirichlet boundary conditions and consider, for small $\eta>0$,

$$
P_{0}^{\eta}(a, b) \begin{cases}-\mathcal{E}\left(v^{\eta}\right)(x, z)-c v_{x}^{\eta}(x, z)=\left(r(z)-\gamma v^{\eta}(x, z)\right) v^{\eta}(x, z) & \text { in } Q \\ v^{\eta}(x, z)=\frac{x-a}{-2 a} \Gamma_{b}^{\delta / 3, \eta}(z) & \text { on } \partial Q\end{cases}
$$

where $\Gamma_{b}^{\delta / 3, \eta}(z):=\Gamma_{b}^{\delta / 3}(z)+\eta \Gamma_{\infty}^{\delta / 3}(z)$. Note that for any nonnegative $\eta, 0$ and a large enough positive constant are respectively a sub- and a super-solution of $P_{0}^{\eta}(a, b)$. Thus the existence of a positive and bounded solution of $P_{0}^{\eta}(a, b)$ can be obtained using a classical monotone iterative scheme. We shall now select $\gamma>0$ so that $(x, z) \mapsto \Gamma_{b}^{\delta / 3, \eta}(z)$ become a super-solution for $P_{0}^{\eta}(a, b)$. Using (15), (16), $\lambda_{b}^{\delta / 3} \geq \lambda_{\infty}^{\delta / 3}$ and $\Gamma_{b}^{\delta / 3, \eta} \geq \Gamma_{b}^{\delta / 3}$ we see that

$$
-\left(B^{2}+1\right) \Delta_{z} \Gamma_{b}^{\delta / 3, \eta}-\left(r(z)-\gamma \Gamma_{b}^{\delta / 3, \eta}\right) \Gamma_{b}^{\delta / 3, \eta} \geq\left(\lambda_{\infty}^{\delta / 3}+\frac{\delta}{3} z^{2}+\gamma \Gamma_{b}^{\delta / 3}\right) \Gamma_{b}^{\delta / 3, \eta},
$$

which is clearly nonnegative for $|z| \geq \bar{z}:=\sqrt{\frac{-3 \lambda_{\infty}^{\delta / 3}}{\delta}}$. Now, as soon as $b \geq \bar{z}+1$, it follows from the Harnack inequality that there is $C>0$ such that $\Gamma_{b}^{\delta / 3}(z) \geq \frac{1}{C} \Gamma_{b}^{\delta / 3}(0)=\frac{1}{C}$ for all $|z| \leq \bar{z}$. Hence if we select $\gamma:=-C \lambda_{\infty}^{\delta / 3}>0$, the right-hand side member of (31) becomes nonnegative for 
$|z| \leq \bar{z}$, and $(x, z) \mapsto \Gamma_{b}^{\delta / 3, \eta}(z)$ is a super-solution for $P_{0}^{\eta}(a, b)$. Next, we show that, any solution of $P_{0}^{\eta}(a, b)$ satisfies

$$
0<v^{\eta}(x, z)<\Gamma_{b}^{\delta / 3, \eta}(z), \quad \forall(x, z) \in Q .
$$

Indeed, for $v^{\eta}$ a non negative solution of $P_{0}^{\eta}(a, b)$, the following quantity is well defined

$$
\alpha_{0}:=\sup \left\{\alpha \geq 0: \forall(x, z) \in \bar{Q}, \alpha v^{\eta}(x, z) \leq \Gamma_{b}^{\delta / 3, \eta}(z)\right\} \in(0,1],
$$

since $\Gamma_{b}^{\delta / 3, \eta}>0$ in $\bar{Q}$. Let us assume by contradiction that $\alpha_{0}<1$. In view of the boundary conditions for $v^{\eta}$, this implies that a point $\left(x_{0}, z_{0}\right)$ where $\alpha_{0} v^{\eta}\left(x_{0}, z_{0}\right)=\Gamma_{b}^{\delta / 3, \eta}\left(z_{0}\right)$ cannot be on $\partial Q$. Hence $w:=\Gamma_{b}^{\delta / 3, \eta}-\alpha_{0} v^{\eta}$ has a zero minimum at $\left(x_{0}, z_{0}\right) \in Q$ and

$$
0 \geq\left(-\mathcal{E}(w)-c w_{x}\right)\left(x_{0}, z_{0}\right) \geq \gamma \alpha_{0}\left(v^{\eta}\right)^{2}\left(x_{0}, z_{0}\right)\left(1-\alpha_{0}\right)>0,
$$

which is absurd. Hence $\alpha_{0}=1$, and $0 \leq v^{\eta} \leq \Gamma_{b}^{\delta / 3, \eta}$ in $\bar{Q}$. Then (32) follows by applying the strong maximum principle.

By the classical sliding method [11], $v^{\eta}$ is strictly decreasing in the $x$ variable. For the convenience of the reader, let us give a proof of this fact. For $h>0$, define $v_{h}^{\eta}(x, z):=v^{\eta}(x+h, z)$. Since $v_{2 a}^{\eta} \equiv 0<v^{\eta}$ thanks to (32), one can define

$$
h^{*}:=\inf \left\{h>0: \forall \tau \in[h, 2 a], v_{\tau}^{\eta} \leq v^{\eta}\right\} .
$$

Assume $h^{*}>0$. Then there are sequences $h_{n} \nearrow h^{*},\left(x_{n}, z_{n}\right)$ with $v_{h_{n}}^{\eta}\left(x_{n}, z_{n}\right)>v^{\eta}\left(x_{n}, z_{n}\right)$. After extraction and using that the infimum $h^{*}$ is achieved, we have a point $\left(x_{\infty}, z_{\infty}\right)$ such that $v_{h^{*}}^{\eta}\left(x_{\infty}, z_{\infty}\right)=v^{\eta}\left(x_{\infty}, z_{\infty}\right)$, i.e. a point of zero maximum for $v_{h^{*}}^{\eta}-v^{\eta}$. Because of the boundary conditions the point $\left(x_{\infty}, z_{\infty}\right)$ cannot lie on the upper or the lower boundary of $\left(-a, a-h^{*}\right) \times(-b, b)$. In view of (32), it is neither allowed to lie on the left or right boundary of $\left(-a, a-h^{*}\right) \times(-b, b)$. Since $v^{\eta}$ and $v_{h^{*}}^{\eta}$ are both solutions of $-\mathcal{E}(v)-c v_{x}=(r(z)-\gamma v) v$ in $\left(-a, a-h^{*}\right) \times(-b, b)$, the maximum principle then yields $v_{h^{*}}^{\eta} \equiv v^{\eta}$, i.e. $v^{\eta}(x, z)=v^{\eta}\left(x+h^{*}, z\right)$. Hence $v^{\eta}(x, z)=v^{\eta}\left(x+n h^{*}, z\right)$ for all $n \geq 0$. Letting $n \rightarrow \infty$ yields $v^{\eta} \equiv 0$, which is a contradiction. It follows that $h^{*}=0$ and $v^{\eta}$ is non increasing in the $x$ variable.

Now, we construct a solution to $P_{0}(a, b)$ as a limit, as $\eta \rightarrow 0$, of solutions to $P_{0}^{\eta}(a, b)$. The interior elliptic estimates imply that, for all $1<p<\infty$, the sequence $\left(v^{\eta}\right)$ is bounded in $W^{2, p}(Q)$. From Sobolev embedding theorem, one can extract a subsequence $\left(v^{\eta}\right)$ converging to some $u$, strongly in $C^{1, \beta}(Q)$ and weakly in $W^{2, p}(Q)$. Moreover, $u$ is a solution of $P_{0}(a, b)$. As a limit of decreasing functions, $u$ is decreasing in the $x$ variable. By differentiating the equation and applying the maximum principle, one then obtains the strict decreasing of $u$ w.r.t. $x$.

It is then standard that, if $\left(c_{1}, u_{1}\right)$ and $\left(c_{2}, u_{2}\right)$ are two solutions of $P_{0}(a, b)$ with $c_{1}>c_{2}$, then $u_{1}<u_{2}$. Indeed, $u_{2}$ is a super-solution of the equation for $\left(c_{1}, u_{1}\right)$. Hence there exists a solution for this equation which is below $u_{2}$. By uniqueness this solution is $u_{1}$. Hence $u_{1} \leq u_{2}$ and, by the strong maximum principle, $u_{1}<u_{2}$. As seen in Lemma 3.5, Lemma 3.6, the solution of $P_{0}(a, b)$ with speed $c=0, c>c^{*}$ satisfy $u(0,0)>\varepsilon^{*}>\varepsilon, u(0,0)<\varepsilon$ respectively, if $a, b$ are large enough. Then, there is a unique $c$, which belongs to $\left(0, c^{*}\right]$, such that the solution $(c, u)$ of $P_{0}(a, b)$ is $\varepsilon$-normalized. The lemma is proved.

In order to apply a Leray-Schauder degree argument in the next subsection, we also need to consider the family $0 \leq \sigma \leq 1$ of local problems

$$
\tilde{P}_{\sigma}(a, b) \begin{cases}-\mathcal{E}(u)(x, z)-c u_{x}(x, z)=(r(z)-(1-\sigma) \mathcal{R}-\sigma \gamma u(x, z)) u(x, z) & \text { in } Q \\ u(x, z)=\mathbf{1}_{\{x=-a\}}(x) \Gamma_{b}^{\delta / 3}(z) & \text { on } \partial Q,\end{cases}
$$

where $\mathcal{R}:=\max _{z \in \mathbb{R}}\left(r(z)+\frac{\delta}{3} z^{2}\right)$.

Lemma 3.8 (On local problems $\tilde{P}_{\sigma}(a, b)$ ) (i) There exists $M>0$ such that, for all $a>0$, $b>0,0 \leq \sigma \leq 1$, any solution $(c, u \geq 0)$ of $\tilde{P}_{\sigma}(a, b)$ satisfies $0 \leq u \leq M$. 
(ii) Let $b>0$ and $\varepsilon \in(0,1)$ be arbitrary. Then there exists $a_{0}=a_{0}(\varepsilon, b)>0$ such that, for all $a \geq a_{0}$, all $0 \leq \sigma \leq 1$, any solution $(c, u \geq 0)$ of $\tilde{P}_{\sigma}(a, b)$ with $c>c^{*}$ satisfies $u(0,0)<\varepsilon$.

(iii) There exists $\varepsilon_{0}>0, a_{0}>0$ such that, for any $a \geq a_{0}$, there exists a speed $-\bar{c}=-\bar{c}(a)<0$ such that for all $b \geq 1$, all $0 \leq \sigma \leq 1$, any solution $(c, u \geq 0)$ of $\tilde{P}_{\sigma}(a, b)$ with $c \leq-\bar{c}$ satisfies $u(0,0)>\varepsilon_{0}$.

(iv) If $a, b$ are large enough and $\varepsilon \in\left(0, \varepsilon_{0}\right)$, then there exists a unique $(c, u \geq 0) \in \mathbb{R} \times W^{2, \infty}(Q)$ solution of

$$
\tilde{P}_{0}(a, b) \begin{cases}-\mathcal{E}(u)(x, z)-c u_{x}(x, z)=(r(z)-\mathcal{R}) u(x, z) & \text { in } Q \\ u(x, z)=\mathbf{1}_{\{x=-a\}}(x) \Gamma_{b}^{\delta / 3}(z) & \text { on } \partial Q\end{cases}
$$

with $u(0,0)=\varepsilon$. Moreover, by the above a priori estimates, $-\bar{c}<c \leq c^{*}$ and $0 \leq u \leq M$.

Proof. Item $(i)$ follows from $-\mathcal{E}(u)-c u_{x} \leq \sigma(\mathcal{R}-\gamma u) u,(18)$ and the maximum principle.

Define

$$
v(x, y):=u\left(\frac{x-B y}{\sqrt{B^{2}+1}}, \sqrt{B^{2}+1} y\right), \quad Q_{1}:=\left\{(x, y):|y|<\frac{b}{\sqrt{B^{2}+1}},\left|\frac{x-B y}{\sqrt{B^{2}+1}}\right|<a\right\} .
$$

Then $v$ is a subsolution of (26) and, to prove $(i i)$, we can reproduce the proof of Lemma 3.5.

Let us prove $($ iii $)$. Observe that $v$ solves

$$
-v_{x x}-v_{y y}-c \sqrt{B^{2}+1} v_{x}=\left(r\left(\sqrt{B^{2}+1} y\right)-(1-\sigma) \mathcal{R}-\sigma \gamma v\right) v \quad \text { in } Q_{1},
$$

that $v(x, y)=0$ for $(x, y) \in \partial Q_{1}$ such that $\frac{x-B y}{\sqrt{B^{2}+1}} \neq-a$, and that $v(x, y)=\Gamma_{b}^{\delta / 3}\left(\sqrt{B^{2}+1} y\right)$ for $(x, y) \in \partial Q_{1}$ such that $\frac{x-B y}{\sqrt{B^{2}+1}}=-a$. It follows from (16) and the Harnack inequality that there exists $C>0$ such that

$$
\Gamma_{b}^{\delta / 3}(z)>C \Gamma_{b}^{\delta / 3}(0)=C, \quad \text { for all } b \geq 1, \text { all }|z| \leq 1 .
$$

Define, for $\alpha>0$,

$$
\psi_{\alpha}(x, y):=\frac{C}{\max _{[-1,1]} \Gamma_{1}^{0}}\left(1-\frac{x+\alpha+\left(a \sqrt{B^{2}+1}+\frac{B}{\sqrt{B^{2}+1}}\right)}{2 a \sqrt{B^{2}+1}}\right) \Gamma_{1}^{0}\left(\sqrt{B^{2}+1} y\right),
$$

in $Q_{2}:=\left\{(x, y):|y|<\frac{1}{\sqrt{B^{2}+1}},\left|\frac{x-B y}{\sqrt{B^{2}+1}}\right|<a\right\}=Q_{1} \cap\left\{|y|<\frac{1}{\sqrt{B^{2}+1}}\right\}$. Since $\psi_{\alpha=2 a \sqrt{B^{2}+1} \leq 0<v}$ in $Q_{2}$, we can define

$$
\alpha_{0}:=\min \left\{\alpha \in\left[0,2 a \sqrt{B^{2}+1}\right]: \forall(x, y) \in \overline{Q_{2}}, \psi_{\alpha}(x, y) \leq v(x, y)\right\} .
$$

Assume by contradiction that $\alpha_{0}>0$. Observe that $\psi_{\alpha_{0}}(x, y)<0=v(x, y)$ for $(x, y) \in \partial Q_{2}$ such that $\frac{x-B y}{\sqrt{B^{2}+1}}=a$ and $|y|<\frac{1}{\sqrt{B^{2}+1}}$, that $\psi_{\alpha_{0}}(x, y)=0<v(x, y)$ for $(x, y) \in \partial Q_{2}$ such that $|y|=\frac{1}{\sqrt{B^{2}+1}}$ and $-a \leq \frac{x-B y}{\sqrt{B^{2}+1}}<a$, and that - by $(36)-\psi_{\alpha_{0}}(x, y)<\Gamma_{b}^{\delta / 3}\left(\sqrt{B^{2}+1} y\right)=v(x, y)$ for $(x, y) \in \partial Q_{2}$ such that $\frac{x-B y}{\sqrt{B^{2}+1}}=-a$. Therefore the only two points on $\partial Q_{2}$ where $v-\psi_{\alpha_{0}}$ may attain its zero minimum value are $\left(\frac{a}{\sqrt{B^{2}+1}} \pm \frac{B}{\sqrt{B^{2}+1}}, \pm \frac{1}{\sqrt{B^{2}+1}}\right)$. But since $\alpha_{0}>0$ we see that, for $\varepsilon>0$ small enough, $\psi_{\alpha_{0}-\varepsilon} \leq 0 \leq v$ in a neighborhood of these two points of the boundary $\partial Q_{2}$. Hence, by the definition of $\alpha_{0}$, there must be a point $\left(x_{0}, y_{0}\right) \in Q_{2}$ where $v-\psi_{\alpha_{0}}$ attains its zero minimum value, so that $0 \geq-\Delta_{x, y}\left(v-\psi_{\alpha_{0}}\right)\left(x_{0}, y_{0}\right)-c \sqrt{B^{2}+1} \partial_{x}\left(v-\psi_{\alpha_{0}}\right)\left(x_{0}, y_{0}\right)$. Using (35), (15) and straightforward computations, we arrive at

$$
\begin{aligned}
0 & \geq v\left(x_{0}, y_{0}\right)\left(-(1-\sigma) \mathcal{R}-\lambda_{1}^{0}-\sigma \gamma v\left(x_{0}, y_{0}\right)\right)-\frac{c}{2 a} \frac{C}{\max \Gamma_{1}^{0}} \Gamma_{1}^{0}\left(\sqrt{B^{2}+1} y_{0}\right) \\
& \geq v\left(x_{0}, y_{0}\right)\left(-\mathcal{R}-\lambda_{1}^{0}-\gamma M\right)-\frac{c}{2 a} \frac{C}{\max \Gamma_{1}^{0}} \Gamma_{1}^{0}\left(\sqrt{B^{2}+1} y_{0}\right) .
\end{aligned}
$$


Since $v\left(x_{0}, y_{0}\right)=\psi_{\alpha_{0}}\left(x_{0}, y_{0}\right) \leq \frac{C}{\max \Gamma_{1}^{0}} \Gamma_{1}^{0}\left(\sqrt{B^{2}+1} y_{0}\right)$ and since $-\mathcal{R}-\lambda_{1}^{0} \leq-\max _{[-1,1]} r-\lambda_{1}^{0} \leq 0$ we end up with

$$
0 \geq \frac{C}{\max \Gamma_{1}^{0}} \Gamma_{1}^{0}\left(\sqrt{B^{2}+1} y_{0}\right)\left(-\mathcal{R}-\lambda_{1}^{0}-\gamma M-\frac{c}{2 a}\right) .
$$

Hence, for large negative speed, namely

$$
c \leq-\bar{c}=-\bar{c}(a):=2 a\left(-\mathcal{R}-\lambda_{1}^{0}-\gamma M\right)<0,
$$

we get a contradiction, so that $\alpha_{0}=0$. It follows that

$$
u(0,0)=v(0,0) \geq \psi_{0}(0,0)=\frac{C}{\max _{[-1,1]} \Gamma_{1}^{0}}\left(\frac{1}{2}-\frac{B}{2 a\left(B^{2}+1\right)}\right)>\frac{C}{3 \max _{[-1,1]} \Gamma_{1}^{0}}=: \varepsilon_{0},
$$

for $a \geq a_{0}$, with $a_{0}>0$ sufficiently large and independent on $b \geq 1$ and $0 \leq \sigma \leq 1$. This concludes the proof of $($ iii).

To prove $(i v)$, observe that, since $-\mathcal{R} \leq \lambda_{\infty}^{\delta / 3}, \Gamma_{b}^{\delta / 3, \eta}(z):=\Gamma_{b}^{\delta / 3}(z)+\eta \Gamma_{\infty}^{\delta / 3}(z)$ is a supersolution for (35). Hence we can reproduce the proof of Lemma 3.7. Notice in particular that if $\left(c_{1}, u_{1}\right)$ and $\left(c_{2}, u_{2}\right)$ are two solutions of $\tilde{P}_{0}(a, b)$ with $c_{1}>c_{2}$, then $u_{1}<u_{2}$.

\subsection{Construction of a solution in the box}

Equipped with a priori estimates of Subsections 3.4 and 3.3, we are now in the position to construct a solution to $P(a, b, \varepsilon)$, with $\varepsilon \in\left(0, \min \left(\varepsilon^{*}, \varepsilon_{0}\right)\right)$. We shall use a Leray-Schauder topological degree argument (see e.g. [10] or [9] for related arguments).

Proposition 3.9 (The solution in a box) Let $\varepsilon \in\left(0, \min \left(\varepsilon^{*}, \varepsilon_{0}\right)\right)$ be arbitrary. There exist $K>0$ and $b_{0}>0$ such that for any $b \geq b_{0}$ the following holds. There exists $a_{0}=a_{0}(b, \varepsilon)$ such that, for all $a \geq a_{0}$, the problem $P(a, b, \varepsilon)$ has a solution $(c, u)$ such that

$$
\|u\|_{C^{2}(Q)} \leq K, \quad 0<c \leq c^{*} .
$$

Proof. For a given nonnegative function $v$ defined on $Q$ and satisfying the Dirichlet boundary conditions as requested in $P(a, b, \varepsilon)$, consider the family $0 \leq \tau \leq 1$ of linear problems

$$
\left(P_{c}^{\tau}\right) \begin{cases}-\mathcal{E}(U)(x, z)-c U_{x}(x, z) & \\ =\left(r(z)-\tau \int_{-b}^{b} k\left(z, z^{\prime}\right) v\left(x, z^{\prime}\right) d z^{\prime}-\gamma(1-\tau) v(x, z)\right) v(x, z) & \text { in } Q \\ U(x, z)=\mathbf{1}_{\{x=-a\}}(x) \Gamma_{b}^{\delta / 3}(z) & \text { on } \partial Q .\end{cases}
$$

Let us define $\mathcal{K}_{\tau}$ the solution operator of the above system. More precisely $\mathcal{K}_{\tau}$ is the mapping of the Banach space $X:=\mathbb{R} \times C^{1, \alpha}(Q)$ - equipped with the norm $\|(c, v)\|_{X}:=\max \left(|c|,\|v\|_{C^{1, \alpha}}\right)$ onto itself defined by

$$
\mathcal{K}_{\tau}:(c, v) \mapsto\left(\varepsilon-v(0,0)+c, U_{c}^{\tau}:=\text { the solution of }\left(P_{c}^{\tau}\right)\right) .
$$

Constructing a solution of $P(a, b, \varepsilon)$ is equivalent to showing that the kernel of $\operatorname{Id}-\mathcal{K}_{1}$ is nontrivial. The operator $\mathcal{K}_{\tau}$ is compact and depends continuously on the parameter $0 \leq \tau \leq 1$. Thus the Leray-Schauder topological argument can be applied. Define the open set

$$
S:=\left\{(c, v): 0<c<c^{*}+1, v>0,\|v\|_{C^{1, \alpha}}<M+1\right\} \subset X,
$$

where $M>0$ is as in Lemma 3.3. It follows from the a priori estimates Lemma 3.3, Lemma 3.5 and Lemma 3.6, that there exists $a_{0}=a_{0}(b, \varepsilon)>0$ such that, for any $a \geq a_{0}, 0 \leq \tau \leq 1$ the operator $\mathrm{Id}-\mathcal{K}_{\tau}$ cannot vanish on the boundary $\partial S$. By the homotopy invariance of the degree we 
thus have $\operatorname{deg}\left(\operatorname{Id}-\mathcal{K}_{1}, S, 0\right)=\operatorname{deg}\left(\operatorname{Id}-\mathcal{K}_{0}, S, 0\right)$. Additionally, thanks to Lemma 3.7, any element of the kernel of Id $-\mathcal{K}_{0}$ belongs to $S$, so that

$$
\operatorname{deg}\left(\operatorname{Id}-\mathcal{K}_{0}, S, 0\right)=\operatorname{deg}\left(\operatorname{Id}-\mathcal{K}_{0}, \tilde{S}, 0\right),
$$

where

$$
\tilde{S}:=\left\{(c, v):-\bar{c}<c<c^{*}+1, v>0,\|v\|_{C^{1, \alpha}}<M+1\right\} \subset X,
$$

with $\bar{c}=\bar{c}(a)>0$ as in Lemma 3.8 (iii).

Let us now consider the family $0 \leq \sigma \leq 1$ of local and linear problems associated with (33), namely

$$
\left(\tilde{P}_{c}^{\sigma}\right) \begin{cases}-\mathcal{E}(U)(x, z)-c U_{x}(x, z)=(r(z)-(1-\sigma) \mathcal{R}-\sigma \gamma v(x, z)) v(x, z) & \text { in } Q \\ U(x, z)=\mathbf{1}_{\{x=-a\}}(x) \Gamma_{b}^{\delta / 3}(z) & \text { on } \partial Q,\end{cases}
$$

and let $\tilde{\mathcal{K}}_{\sigma}$ be the associated solution operator, that is

$$
\tilde{\mathcal{K}}_{\sigma}:(c, v) \mapsto\left(\varepsilon-v(0,0)+c, \tilde{U}_{c}^{\sigma}:=\text { the solution of }\left(\tilde{P}_{c}^{\sigma}\right)\right) .
$$

The operator $\tilde{\mathcal{K}}_{\sigma}$ is compact and depends continuously on the parameter $0 \leq \sigma \leq 1$. The analysis of the local problems $\tilde{P}_{\sigma}(a, b)$ in Lemma 3.8 shows that Id $-\tilde{\mathcal{K}}_{\sigma}$ cannot vanish on the boundary of $\tilde{S}$. Since $\mathcal{K}_{0}=\tilde{\mathcal{K}}_{1}$ we have

$$
\operatorname{deg}\left(\operatorname{Id}-\mathcal{K}_{0}, \tilde{S}, 0\right)=\operatorname{deg}\left(\operatorname{Id}-\tilde{\mathcal{K}}_{0}, \tilde{S}, 0\right) .
$$

To complete the proof, let us compute $\operatorname{deg}\left(\mathrm{Id}-\tilde{\mathcal{K}}_{0}, \tilde{S}, 0\right)$ by using two additional homotopies. First, consider, for $0 \leq \tau \leq 1$,

$$
\mathcal{G}_{\tau}:(c, v) \mapsto\left(\varepsilon-(1-\tau) v(0,0)-\tau \tilde{U}_{c}^{0}(0,0)+c, \tilde{U}_{c}^{0}:=\text { the solution of }\left(\tilde{P}_{c}^{0}\right)\right) .
$$

If $\mathcal{G}_{\tau}(c, v)=(c, v)$ for some $(c, v) \in \partial \tilde{S}$, then $(c, v) \in \partial \tilde{S}$ solves the local problem $\tilde{P}^{0}(a, b)$ and is such that $v(0,0)=\varepsilon$. By the a priori estimates of Lemma 3.8, this cannot be. Therefore Id $-\mathcal{G}_{\tau}$ does not vanish on the boundary $\partial \tilde{S}$. Since $\tilde{\mathcal{K}}_{0}=\mathcal{G}_{0}$ we have $\operatorname{deg}\left(\operatorname{Id}-\tilde{\mathcal{K}}_{0}, \tilde{S}, 0\right)=$ $\operatorname{deg}\left(\operatorname{Id}-\mathcal{G}_{1}, \tilde{S}, 0\right)$. Next, we know from Lemma $3.8(i v)$ that there is a unique $\left(c_{0}, \tilde{U}_{c_{0}}\right) \in \tilde{S}$ which solves the local problem $\tilde{P}^{0}(a, b)$ and is such that $\tilde{U}_{c_{0}}(0,0)=\varepsilon$. Then, consider, for $0 \leq \tau \leq 1$,

$$
\mathcal{H}_{\tau}:(c, v) \mapsto\left(\varepsilon-\tilde{U}_{c}^{0}(0,0)+c, \tau \tilde{U}_{c}^{0}+(1-\tau) \tilde{U}_{c_{0}}\right) .
$$

If $\mathcal{H}_{\tau}(c, v)=(c, v)$ for some $(c, v) \in \partial \tilde{S}$, then the uniqueness in Lemma $3.8(i v)$ enforces $c=c_{0}$, $\tilde{U}_{c}^{0}=\tilde{U}_{c_{0}}=v$ so that $(c, v) \in \partial \tilde{S}$ solves the local problem $\tilde{P}^{0}(a, b)$ and is such that $v(0,0)=\varepsilon$, which cannot be. Therefore $\operatorname{Id}-\mathcal{H}_{\tau}$ does not vanish on the boundary $\partial \tilde{S}$. Since $\mathcal{H}_{1}=\mathcal{G}_{1}$ we have $\operatorname{deg}\left(\operatorname{Id}-\mathcal{G}_{1}, \tilde{S}, 0\right)=\operatorname{deg}\left(\operatorname{Id}-\mathcal{H}_{0}, \tilde{S}, 0\right)$, where

$$
\mathrm{Id}-\mathcal{H}_{0}:(c, v) \mapsto\left(\tilde{U}_{c}^{0}(0,0)-\varepsilon, v-\tilde{U}_{c_{0}}\right) .
$$

As seen in the proof of Lemma $3.8(i v), \tilde{U}_{c}^{0}(0,0)$ is strictly decreasing in $c$ so the degree of the first component of the above operator is -1 . Clearly the degree of the second one is 1 . Hence $\operatorname{deg}\left(\operatorname{Id}-\mathcal{H}_{0}, \tilde{S}, 0\right)=-1$ so that $\operatorname{deg}\left(\operatorname{Id}-\mathcal{K}_{1}, S, 0\right)=-1$ and there is a $(c, u) \in S$ solution of $P(a, b, \varepsilon)$. This concludes the proof of the proposition. 


\section{The front with minimal speed $c^{*}$}

Equipped with the solution $(c, u)$ of $P(a, b, \varepsilon)$, with $\varepsilon \in\left(0, \min \left(\varepsilon^{*}, \varepsilon_{0}\right)\right)$, of Proposition 3.9 , we now let $a \rightarrow+\infty$. Note that we have the bounds (37) on $c$ and $u$, and also the Gaussian control of the tails in Lemma 3.4. This enables to construct — passing to a subsequence $a_{n} \rightarrow+\infty$ - a speed $0 \leq c_{b} \leq c^{*}$ and a function $u_{b} \in C_{b}^{2}(\mathbb{R} \times[-b, b])$ with the same bounds as those of $u$. Similarly, we can then consider $b \rightarrow+\infty$, to construct, via a subsequence $b_{n} \rightarrow+\infty$, a speed $0 \leq c \leq c^{*}$ and a function $u \in C_{b}^{2}\left(\mathbb{R}^{2}\right)$, such that $0<u \leq K$, and

$$
\begin{gathered}
-\mathcal{E}(u)(x, z)-c u_{x}(x, z)=\left(r(z)-\int_{\mathbb{R}} k\left(z, z^{\prime}\right) u\left(x, z^{\prime}\right) d z^{\prime}\right) u(x, z) \quad \text { in } \mathbb{R}^{2} \\
u(0,0)=\varepsilon \\
0 \leq u(x, z) \leq \bar{M} \Gamma_{\infty}^{2 \delta / 3}(z), \quad \forall(x, z) \in \mathbb{R}^{2} .
\end{gathered}
$$

\subsection{The constructed wave has the minimal speed $c^{*}$}

Here, we show that, by reducing the normalization (40) if necessary, the above constructed solution has speed $c=c^{*}$.

Lemma 4.1 (A priori estimate for the infimum) There exists $\varepsilon>0$ such that any solution $(c, u)$ of (39), (41) with $c \geq 0$ and $\inf _{x \in \mathbb{R}} u(x, 0)>0$ actually satisfies $\inf _{x \in \mathbb{R}} u(x, 0)>\varepsilon$.

Proof. We choose $R>0$ large enough, such that

$$
\lambda_{\infty}^{0} \leq \lambda_{R}^{0}<\frac{\lambda_{\infty}^{0}}{2}<0, \quad k^{+} \int_{[-R, R]^{c}} \bar{M} \Gamma_{\infty}^{2 \delta / 3}(z) d z \leq \frac{-\lambda_{\infty}^{0}}{4} .
$$

Thanks to the Harnack inequality, there exists $C>0$ such that

$$
u\left(x, z^{\prime}\right) \leq C u(x, z) \quad \text { for all } x \in \mathbb{R},|z| \leq R,\left|z^{\prime}\right| \leq R,
$$

which, combined with (41) and the second part of (42), implies $\int k\left(z, z^{\prime}\right) u\left(x, z^{\prime}\right) d z^{\prime} \leq \frac{-\lambda_{\infty}^{0}}{4}+$ $2 k^{+} C R u(x, z)$ in the $\operatorname{strip} \mathbb{R} \times(-R, R)$. Hence, $-\mathcal{E}(u)-c u_{x} \geq\left(r(z)-\left(\frac{-\lambda_{\infty}^{0}}{4}+2 k^{+} C R u\right)\right) u$ in $\mathbb{R} \times(-R, R)$. Changing variables, the function $v(x, y):=u\left(\frac{x-B y}{\sqrt{B^{2}+1}}, \sqrt{B^{2}+1} y\right)$ then satisfies

$$
-v_{x x}-v_{y y}-c \sqrt{B^{2}+1} v_{x} \geq\left(r\left(\sqrt{B^{2}+1} y\right)-\left(\frac{-\lambda_{\infty}^{0}}{4}+2 k^{+} C R v\right)\right) v
$$

in $S:=\mathbb{R} \times\left(-\frac{R}{\sqrt{B^{2}+1}}, \frac{R}{\sqrt{B^{2}+1}}\right)$.

Now let $\eta>0$ be arbitrarily given. Define, for $\alpha>0$,

$$
\psi_{\alpha}(x, y):=\alpha\left(1-\eta x^{2}\right) \Gamma_{R}^{0}\left(\sqrt{B^{2}+1} y\right) .
$$

Observe that the Harnack inequality (43) implies $\psi_{\alpha} \leq v$ for $\alpha=C^{-1}\left\|\Gamma_{R}^{0}\right\|_{\infty}^{-1} \inf _{x \in \mathbb{R}} u(x, 0)$, and that (41) implies $\psi_{\alpha}(0,0)=\bar{M} \geq v(0,0)$ for $\alpha=\bar{M}$. We can therefore define

$$
\alpha_{0}:=\max \left\{\alpha>0: \forall(x, y) \in \bar{S}, \psi_{\alpha}(x, y) \leq v(x, y)\right\} \in(0, \bar{M}] .
$$

Hence $v-\psi_{\alpha_{0}}$ attains a zero minimum at a point $\left(x_{0}, y_{0}\right)$ - depending on $\eta$ - which must lie in $\left(-\frac{1}{\sqrt{\eta}}, \frac{1}{\sqrt{\eta}}\right) \times\left(-\frac{R}{\sqrt{B^{2}+1}}, \frac{R}{\sqrt{B^{2}+1}}\right)$ so that

$$
0 \geq-\Delta_{x, y}\left(v-\psi_{\alpha_{0}}\right)\left(x_{0}, y_{0}\right)-c \sqrt{B^{2}+1} \partial_{x}\left(v-\psi_{\alpha_{0}}\right)\left(x_{0}, y_{0}\right)
$$


Using (44) and straightforward computations, we arrive at

$$
0 \geq\left(\frac{\lambda_{\infty}^{0}}{4}-2 k^{+} C R v\left(x_{0}, y_{0}\right)-\lambda_{R}^{0}\right) v\left(x_{0}, y_{0}\right)-2 \alpha_{0}\left(\eta+c \sqrt{B^{2}+1} \eta x_{0}\right) \Gamma_{R}^{0}\left(\sqrt{B^{2}+1} y_{0}\right) .
$$

Using the first part of (42), $\alpha_{0} \leq \bar{M}$ and $\left|x_{0}\right| \leq \frac{1}{\sqrt{\eta}}$, this yields

$$
0 \geq\left(\frac{-\lambda_{\infty}^{0}}{4}-2 k^{+} C R v\left(x_{0}, y_{0}\right)\right) v\left(x_{0}, y_{0}\right)-2 \bar{M}\left\|\Gamma_{R}^{0}\right\|_{\infty}\left(\eta+c \sqrt{B^{2}+1} \sqrt{\eta}\right) .
$$

It follows from the Harnack inequality (43) that $v\left(x_{0}, y_{0}\right) \geq \frac{1}{C} \inf _{x \in \mathbb{R}} u(x, 0)>0$, so that

$$
v\left(x_{0}, y_{0}\right) \geq \frac{1}{2 k^{+} C R}\left(\frac{-\lambda_{\infty}^{0}}{4}-\frac{2 C \bar{M}\left\|\Gamma_{R}^{0}\right\|_{\infty}\left(\eta+c \sqrt{B^{2}+1} \sqrt{\eta}\right)}{\inf _{x \in \mathbb{R}} u(x, 0)}\right) .
$$

Since $\eta>0$ can be chosen arbitrarily small, we have $v\left(x_{0}, y_{0}\right) \geq \frac{-\lambda_{\infty}^{0}}{8 k^{+} C R}$ and then $\alpha_{0} \geq \varepsilon:=$ $\frac{-\lambda_{\infty}^{0}}{8 k^{+} C R\left\|\Gamma_{R}^{0}\right\|_{\infty}}>0$. Hence, $v(x, y) \geq \varepsilon\left(1-\eta x^{2}\right) \Gamma_{R}^{0}\left(\sqrt{B^{2}+1} y\right)$ for all $(x, y) \in \bar{S}$. Since $\eta>0$ can be chosen arbitrarily small, we have $v(x, y) \geq \frac{\varepsilon}{2} \Gamma_{R}^{0}\left(\sqrt{B^{2}+1} y\right)$, and in particular $\inf _{x \in \mathbb{R}} u(x, 0)=$ $\inf _{x \in \mathbb{R}} v(x, 0) \geq \frac{\varepsilon}{2}$. This proves the lemma.

As a result, the constructed solution of (39), (40), (41) satisfies $\inf _{x \in \mathbb{R}} u(x, 0)=0$. Without loss of generality, we may assume $\liminf _{x \rightarrow \infty} u(x, 0)=0$. The following proposition then enforces $c=c^{*}$ for the constructed wave. It is also of independent interest since it proves the non existence of waves for $0 \leq c<c^{*}$ as stated in Theorem 1.4 (ii).

Proposition $4.2\left(c=c^{*}\right.$ for the constructed wave) Any solution $(c, u)$ of (39), (41) with $c \geq$ 0 and $\liminf _{x \rightarrow \infty} u(x, 0)=0$ actually satisfies $c \geq c^{*}$.

Proof. Assume by contradiction that $0 \leq c<c^{*}$. Choose $c<\tilde{c}<c^{*}$. Since $\lambda_{R}^{0} \rightarrow \lambda_{\infty}^{0}=$ $-\left(B^{2}+1\right) \frac{c^{* 2}}{4}$ as $R \rightarrow \infty$, we can choose $R>0$ such that

$$
\frac{\lambda_{R}^{0}}{B^{2}+1} \leq-\frac{\tilde{c}^{2}}{4}-\frac{1}{2} \frac{c^{* 2}-\tilde{c}^{2}}{4}, \quad k^{+} \int_{[-R, R]^{c}} \bar{M} \Gamma_{\infty}^{2 \delta / 3} \leq \frac{B^{2}+1}{4} \frac{c^{* 2}-\tilde{c}^{2}}{4} .
$$

Let us define the open rectangle

$$
\Omega:=\left\{(x, y):|x| \leq \bar{x}:=\frac{\pi}{\sqrt{\left(B^{2}+1\right)\left(\tilde{c}^{2}-c^{2}\right)}},|y| \leq \bar{y}:=\frac{R}{\sqrt{B^{2}+1}}\right\} .
$$

Thanks to the Harnack inequality, there exists $C>0$ such that for any solution $(c, u)$ of $(39),(41)$ with $0 \leq c \leq c^{*}$, and for all $x_{1} \in \mathbb{R}$

$$
\max _{\left(x, y, z^{\prime}\right) \in \bar{\Omega} \times[-R, R]} u\left(x_{1}+\frac{x-B y}{\sqrt{B^{2}+1}}, z^{\prime}\right) \leq C u\left(x_{1}, 0\right) .
$$

Following the change of variables of Lemma 3.5 , we see that $v(x, y):=u\left(\frac{x-B y}{\sqrt{B^{2}+1}}, \sqrt{B^{2}+1} y\right)$ satisfies

$$
\begin{aligned}
-v_{x x}-v_{y y}-c \sqrt{B^{2}+1} v_{x} & =\left[r\left(\sqrt{B^{2}+1} y\right)-\int k\left(\sqrt{B^{2}+1} y, z^{\prime}\right) u\left(\frac{x-B y}{\sqrt{B^{2}+1}}, z^{\prime}\right) d z^{\prime}\right] v \\
& \geq\left[r\left(\sqrt{B^{2}+1} y\right)-\left(\frac{B^{2}+1}{4} \frac{c^{* 2}-\tilde{c}^{2}}{4}+k^{+} 2 C R u(0,0)\right)\right] v,
\end{aligned}
$$


where we have used the second inequality in (45) and (46) with $x_{1}=0$. Next, define the function

$$
\psi(x, y):=\Gamma_{R}^{0}\left(\sqrt{B^{2}+1} y\right) e^{-\frac{c \sqrt{B^{2}+1} x}{2}} \sin \left(\frac{\sqrt{\left(B^{2}+1\right)\left(\tilde{c}^{2}-c^{2}\right)}}{2} x+\frac{\pi}{2}\right) .
$$

We have $\psi=0$ on $\partial \Omega$ and, using the first inequality in (45),

$$
-\psi_{x x}-\psi_{y y}-c \sqrt{B^{2}+1} \psi_{x}-r\left(\sqrt{B^{2}+1} y\right) \psi=\left(\left(B^{2}+1\right) \frac{\tilde{c}^{2}}{4}+\lambda_{R}^{0}\right) \psi \leq-\frac{B^{2}+1}{2} \frac{c^{* 2}-\tilde{c}^{2}}{4} \psi,
$$

in $\Omega$.

Since $0<v \leq K$, we can define $\alpha_{0}:=\max \{\alpha>0: \alpha \psi \leq v$ in $\bar{\Omega}\}>0$ and there is a point $\left(x_{0}, y_{0}\right) \in \Omega$ where $w:=\alpha_{0} \psi-v$ attains a zero maximum. In view of the above inequalities, we have at point $\left(x_{0}, y_{0}\right)$,

$$
\begin{aligned}
0 \leq-w_{x x}-w_{y y}-c \sqrt{B^{2}+1} w_{x}-r\left(\sqrt{B^{2}+1} y\right) w \\
\leq\left[-\frac{B^{2}+1}{4} \frac{c^{* 2}-\tilde{c}^{2}}{4}+k^{+} 2 C R u(0,0)\right] v\left(x_{0}, y_{0}\right),
\end{aligned}
$$

which in turn implies $u(0,0) \geq\left(B^{2}+1\right) \frac{c^{* 2}-\tilde{c}^{2}}{32 k^{+} C R}$. In view of (46), the argument is invariant under translation w.r.t. $x$ variable, so that

$$
\inf _{x \in \mathbb{R}} u(x, 0) \geq\left(B^{2}+1\right) \frac{c^{* 2}-\tilde{c}^{2}}{32 k^{+} C R}>0,
$$

which contradicts $\lim \inf _{x \rightarrow \infty} u(x, 0)=0$. The proposition is proved.

\subsection{Behaviors as $x \rightarrow \pm \infty$}

The following proposition will show that the constructed wave satisfies the lower bound in (11) and (12) in Theorem 1.4.

Proposition 4.3 (Behaviors at infinity) Let (c,u) be a solution of (39), (40), (41) with $c \geq 0$. Then the following holds.

(i) There exist $R>0$ and $\kappa>0$ such that $u(x, z) \geq \kappa \Gamma_{R}^{0}(z)$ for all $(x, z) \in(-\infty, 0] \times[-R, R]$.

(ii) If $\varepsilon>0$ is as in Lemma 4.1, then both $\int_{\mathbb{R}} u(x, z) d z \rightarrow 0$ and $\max _{z \in \mathbb{R}} u(x, z) \rightarrow 0$, as $x \rightarrow \infty$.

Proof. Let us prove (i). We start as in the proof of Lemma 4.1: choose $R>0$ large enough so that (42) holds, choose $C>0$ such that both (43) and

$$
\min _{-\frac{1}{2} \leq x \leq 0,|y| \leq \frac{R}{\sqrt{B^{2}+1}}} v(x, y) \geq \frac{1}{C} u(0,0)=\frac{\varepsilon}{C}
$$

hold, and observe that $v(x, y):=u\left(\frac{x-B y}{\sqrt{B^{2}+1}}, \sqrt{B^{2}+1} y\right)$ satisfies (44) in the strip $S:=\mathbb{R} \times$ $\left(-\frac{R}{\sqrt{B^{2}+1}}, \frac{R}{\sqrt{B^{2}+1}}\right)$. Now for $\eta>0$, we define

$$
\psi_{\eta}(x, y):=\alpha\left(\frac{1}{2}+\eta x\right) \Gamma_{R}^{0}\left(\sqrt{B^{2}+1} y\right), \quad \alpha:=\min \left(\frac{\varepsilon}{C\left\|\Gamma_{R}^{0}\right\|_{\infty}}, \frac{-\lambda_{\infty}^{0}}{5 k^{+} C R\left\|\Gamma_{R}^{0}\right\|_{\infty}}\right) .
$$

The definition of $\alpha$ then enforces $\psi_{1} \leq v$ on $\overline{S_{-}}$, where $S_{-}:=(-\infty, 0) \times\left(-\frac{R}{\sqrt{B^{2}+1}}, \frac{R}{\sqrt{B^{2}+1}}\right)$. We can therefore define

$$
\eta_{0}:=\min \left\{\eta \geq 0: \forall(x, y) \in \overline{S_{-}}, \psi_{\eta}(x, y) \leq v(x, y)\right\} \in[0,1] .
$$


Let us assume by contradiction that $\eta_{0}>0$. Function $v-\psi_{\eta_{0}}$ then attains a zero minimum at a point $\left(x_{0}, y_{0}\right)$; the definition of $\alpha$ and the Harnack inequality (48) prevents $x_{0}=0$ so that $\left(x_{0}, y_{0}\right)$ has to lie in $\left(-\frac{1}{2 \eta_{0}}, 0\right) \times\left(-\frac{R}{\sqrt{B^{2}+1}}, \frac{R}{\sqrt{B^{2}+1}}\right)$. We therefore have $0 \geq-\Delta_{x, y}\left(v-\psi_{\eta_{0}}\right)\left(x_{0}, y_{0}\right)-$ $c \sqrt{B^{2}+1} \partial_{x}\left(v-\psi_{\eta_{0}}\right)\left(x_{0}, y_{0}\right)$. Using (44), $\partial_{x} \psi_{\eta_{0}} \geq 0$ and the first part of (42) we arrive at

$$
0 \geq\left(\frac{-\lambda_{\infty}^{0}}{4}-2 k^{+} C R v\left(x_{0}, y_{0}\right)\right) v\left(x_{0}, y_{0}\right)
$$

which in turn implies $\frac{-\lambda_{\infty}^{0}}{8 k^{+} C R} \leq v\left(x_{0}, y_{0}\right)=\psi_{\eta_{0}}\left(x_{0}, y_{0}\right) \leq \frac{\alpha}{2}\left\|\Gamma_{R}^{0}\right\|_{\infty}$, which contradicts the definition of $\alpha$. Hence $\eta_{0}=0$ and $v(x, y) \geq \frac{\alpha}{2} \Gamma_{R}^{0}\left(\sqrt{B^{2}+1} y\right)$ for all $(x, y) \in(-\infty, 0] \times$ $\left[-\frac{R}{\sqrt{B^{2}+1}}, \frac{R}{\sqrt{B^{2}+1}}\right]$. This concludes the proof of $(i)$.

Thanks to the Harnack inequality and the control of the tails $u(x, z) \leq \bar{M} \Gamma_{\infty}^{2 \delta / 3}(z)$, in order to prove $(i i)$ it is enough to prove $u(x, 0) \rightarrow$, as $x \rightarrow \infty$. Assume by contradiction that there exists $\nu>0$ and $x_{n} \rightarrow+\infty$ such that $u\left(x_{n}, 0\right) \geq \nu$, for all $n$. Then, the proof of $(i)$ shows that $u(x, 0) \geq \frac{1}{2} \min \left(\frac{\nu}{C\left\|\Gamma_{R}^{0}\right\|_{\infty}}, \frac{-\lambda_{\infty}^{0}}{5 k^{+} C R\left\|\Gamma_{R}^{0}\right\|_{\infty}}\right)$ for all $x \in\left(-\infty, x_{n}\right)$, and then for all $x \in \mathbb{R}$.

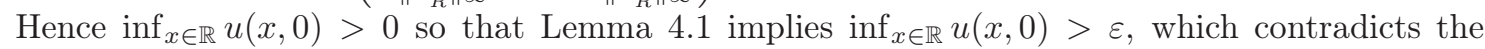
normalization (40). This proves $(i i)$.

\section{$5 \quad$ Faster fronts $\left(c>c^{*}\right)$}

In this section we fix $c>c^{*}=2 \sqrt{\frac{-\lambda_{\infty}^{0}}{B^{2}+1}}$ and construct a nonnegative function $u \in C^{2}\left(\mathbb{R}^{2}\right)$ solution of

$$
-\mathcal{E}(u)(x, z)-c u_{x}(x, z)=\left(r(z)-\int_{\mathbb{R}} k\left(z, z^{\prime}\right) u\left(x, z^{\prime}\right) d z^{\prime}\right) u(x, z) \quad \text { in } \mathbb{R}^{2} .
$$

Using the change of variables $v(x, y):=u\left(\frac{x-B y}{\sqrt{B^{2}+1}}, \sqrt{B^{2}+1} y\right)$, we need to construct a nonnegative $v=v(x, y)$ solution of

$$
\begin{aligned}
\mathcal{L} v(x, y) & :=-v_{x x}(x, y)-v_{y y}(x, y)-c \sqrt{B^{2}+1} v_{x}(x, y)-r\left(\sqrt{B^{2}+1} y\right) v(x, y)= \\
& -v(x, y) \int_{\mathbb{R}} k\left(\sqrt{B^{2}+1} y, z^{\prime}\right) v\left(x-B y+\frac{B}{\sqrt{B^{2}+1}} z^{\prime}, \frac{z^{\prime}}{\sqrt{B^{2}+1}}\right) d z^{\prime} \quad \text { in } \mathbb{R}^{2} .
\end{aligned}
$$

Note also that solving the problem in the box

$$
-\mathcal{E}(u)(x, z)-c u_{x}(x, z)=\left(r(z)-\int_{-b}^{b} k\left(z, z^{\prime}\right) u\left(x, z^{\prime}\right) d z^{\prime}\right) u(x, z) \quad \text { in } Q=(-a, a) \times(-b, b),
$$

is equivalent to solving

$$
\mathcal{L} v(x, y)=-v(x, y) \int_{-b}^{b} k\left(\sqrt{B^{2}+1} y, z^{\prime}\right) v\left(x-B y+\frac{B}{\sqrt{B^{2}+1}} z^{\prime}, \frac{z^{\prime}}{\sqrt{B^{2}+1}}\right) d z^{\prime} \quad \text { in } Q_{1},
$$

with $Q_{1}=\left\{(x, y):|y|<\frac{b}{\sqrt{B^{2}+1}},\left|\frac{x-B y}{\sqrt{B^{2}+1}}\right|<a\right\}$.

We first adapt the strategy of [9]: we construct a solution in the box by using sub and supersolutions and the Schauder fixed point theorem. This will allow to let $a \rightarrow \infty$ but we shall need a extra argument to let $b \rightarrow \infty$.

Construction of sub and supersolutions. We use again the supersolution of Lemma 3.5: since $c>c^{*}$, one can select $\mu<0$ the largest root of $\mu^{2}+c \sqrt{B^{2}+1} \mu+\frac{c^{* 2}}{4}\left(B^{2}+1\right)=0$. Then the function

$$
w(x, y):=e^{\mu x} \Gamma_{\infty}^{0}\left(\sqrt{B^{2}+1} y\right)
$$


with $\Gamma_{\infty}^{0}$ the eigenfunction appearing in Definition 1.2, satisfies $\mathcal{L} w(x, y)=0$ in $\mathbb{R}^{2}$.

Next, we aim at constructing a kind of subsolution. Precisely we look after a function $h$ such that (note that the supersolution $w$ appears in the integral term)

$\mathcal{L} h(x, y) \leq-h(x, y) \int_{-b}^{b} k\left(\sqrt{B^{2}+1} y, z^{\prime}\right) w\left(x-B y+\frac{B}{\sqrt{B^{2}+1}} z^{\prime}, \frac{z^{\prime}}{\sqrt{B^{2}+1}}\right) d z^{\prime} \quad$ in $\{h>0\}$.

Since $k \leq k^{+}$and $\int_{\mathbb{R}} e^{\mu \frac{B}{\sqrt{B^{2}+1}} z^{\prime}} \Gamma_{\infty}^{0}\left(z^{\prime}\right) d z^{\prime}<\infty$, there is $C>0$ such that

$$
\int_{\mathbb{R}} k\left(\sqrt{B^{2}+1} y, z^{\prime}\right) w\left(x-B y+\frac{B}{\sqrt{B^{2}+1}} z^{\prime}, \frac{z^{\prime}}{\sqrt{B^{2}+1}}\right) d z^{\prime} \leq C e^{\mu(x-B y)} .
$$

Let us choose $\varepsilon>0$ small enough so that $-\rho:=(\mu-\varepsilon)^{2}+c \sqrt{B^{2}+1}(\mu-\varepsilon)+\frac{c^{* 2}}{4}\left(B^{2}+1\right)<0$ and $\mu+\varepsilon<0$. For a constant $A>1$ to be selected later, let us define

$$
h(x, y):=\left(\frac{1}{A} e^{\mu x}-e^{(\mu-\varepsilon) x}\right) \Gamma_{\infty}^{0}\left(\sqrt{B^{2}+1} y\right)=\frac{1}{A} w(x, y)-e^{(\mu-\varepsilon) x} \Gamma_{\infty}^{0}\left(\sqrt{B^{2}+1} y\right),
$$

with is nonnegative if and only if $x \geq \varepsilon^{-1} \ln A$. Thanks to the estimate (54), we have

$$
\begin{aligned}
& \mathcal{L} h(x, y)+h(x, y) \int_{-b}^{b} k\left(\sqrt{B^{2}+1} y, z^{\prime}\right) w\left(x-B y+\frac{B}{\sqrt{B^{2}+1}} z^{\prime}, \frac{z^{\prime}}{\sqrt{B^{2}+1}}\right) d z^{\prime} \\
& \leq\left(-\rho+C e^{\mu(x-B y)}\right) h(x, y) \\
& \leq 0
\end{aligned}
$$

in $\left\{(x, y) \in Q_{1}: x \geq \frac{1}{\mu} \ln \left(\frac{\rho}{C}\right)+\frac{B}{\sqrt{B^{2}+1}} b\right\}$ which contains $\{h>0\}=\left\{(x, y) \in Q_{1}: x \geq \varepsilon^{-1} \ln A\right\}$ provided that $A$ is chosen sufficiently large. Notice that $A$ does not depend on $a$ but does depend on $b$. We will thus need an extra argument below.

Construction of a propagating wave in the strip $\mathbb{R} \times[-b, b]$. Let $b>0$ be arbitrary. Consider the problem (52) in the box $Q_{1}$ supplemented with the boundary conditions

$$
v(x, y)=h_{0}(x, y):=\max (0, h(x, y)) \quad \text { for all }(x, y) \in \partial Q_{1} .
$$

Define the convex set of functions

$$
R_{a, b}:=\left\{v \in C\left(Q_{1}\right): h_{0} \leq v \leq w\right\},
$$

and the compact application $\Phi_{a, b}$ that maps a given $v^{\star} \in C\left(Q_{1}\right)$ to the solution $v$ of

$$
\mathcal{L} v(x, y)=-v(x, y) \int_{-b}^{b} k\left(\sqrt{B^{2}+1} y, z^{\prime}\right) v^{\star}\left(x-B y+\frac{B}{\sqrt{B^{2}+1}} z^{\prime}, \frac{z^{\prime}}{\sqrt{B^{2}+1}}\right) d z^{\prime} \quad \text { in } Q_{1},
$$

supplemented with (56). Since $\mathcal{L} v \leq 0=\mathcal{L} w$ in $Q_{1}, v=h_{0} \leq w$ on $\partial Q_{1}$, the maximum principle implies $v \leq w$ in $Q_{1}$. Also, (55) implies $\mathcal{L} h_{0}+h_{0} \int k w \leq 0=\mathcal{L} v+v \int k v^{\star} \leq \mathcal{L} v+v \int k w$ in $Q_{1} \cap\{h>0\}$, and $v \geq 0$ implies $h_{0} \leq v$ on $\partial\left(Q_{1} \cap\{h>0\}\right)$. Hence we have $h_{0} \leq v$ in $Q_{1} \cap\{h>0\}$ and thus in $Q_{1}$. Hence, $\Phi_{a, b}$ maps $R_{a, b}$ into itself. By the Schauder fixed point theorem, $\Phi_{a, b}$ has a fixed point $v_{a, b} \in R_{a, b}$ which solves the problem in the box (52) and satisfies the boundary conditions (56). Hence we are equipped with $u_{a, b}$ solution of (51) with

$$
u_{a, b}(x, z)=h_{0}\left(\sqrt{B^{2}+1} x+\frac{B}{\sqrt{B^{2}+1}} z, \frac{z}{\sqrt{B^{2}+1}}\right) \quad \text { on } \partial Q
$$

and

$$
h_{0}\left(\sqrt{B^{2}+1} x+\frac{B}{\sqrt{B^{2}+1}} z, \frac{z}{\sqrt{B^{2}+1}}\right) \leq u_{a, b}(x, z) \leq e^{\mu\left(\sqrt{B^{2}+1} x+\frac{B}{\sqrt{B^{2}+1}} z\right)} \Gamma_{\infty}^{0}(z) \quad \text { in } \bar{Q} .
$$


We claim (see proof below) that there exists $\bar{M}>0$ such that, for all $a>0, b>0$,

$$
u_{a, b}(x, z) \leq \bar{M} \Gamma_{\infty}^{2 \delta / 3}(z) \leq \bar{M}\left\|\Gamma_{\infty}^{2 \delta / 3}\right\|_{\infty}=: M, \quad \text { in } \bar{Q} .
$$

Now, for a given $b>0$, choose $A=A_{b}$ as in the construction of $h$ above. The family $\left(u_{a, b}\right)_{a}$ is uniformly bounded, and is then uniformly bounded in $C^{2, \alpha}(Q)$. This allows to let $a \rightarrow \infty$, possibly along a subsequence. In this limit, we have $u_{a, b} \rightarrow u_{b}$, which is a solution of (49) in the strip $S_{b}:=\mathbb{R} \times(-b, b)$, and satisfies $(58),(59)$ in $\overline{S_{b}}$; in particular (58) yields

$$
u_{b}\left(x_{b}, 0\right) \geq h_{0}\left(\frac{1}{\varepsilon} \ln \left(\frac{\mu-\varepsilon}{\mu} A_{b}\right)\right)=: \varepsilon_{b}>0, \quad x_{b}:=\frac{1}{\sqrt{B^{2}+1}} \frac{1}{\varepsilon} \ln \left(\frac{\mu-\varepsilon}{\mu} A_{b}\right) .
$$

Since the problem in the strip is invariant w.r.t. translation in the $x$ variable, we may assume $u_{b}(0,0) \geq \varepsilon_{b}>0$. Since $\varepsilon_{b} \rightarrow 0$ as $b \rightarrow \infty$, before letting $b \rightarrow \infty$ we need an additional argument to get a uniform w.r.t. $b$ lower bound for $u_{b}(0,0)$.

Let us now prove (59). For $0 \leq x \leq a$, it follows from (58) that the mass satisfies $m(x):=$ $\int_{-b}^{b} u_{a, b}(x, z) d z \leq C:=\int_{\mathbb{R}} e^{\mu \frac{B}{\sqrt{B^{2}+1}} z} \Gamma_{\infty}^{0}(z) d z$. For $-a \leq x \leq 0$, observe that $u_{a, b}=h_{0}=0$ on $\partial((-a, 0) \times(-b, b))$ so that we can follow Lemma 3.2 to obtain that the mass satisfies a Fisher-KPP inequality. Since $m(-a)=0$ and $m(0) \leq C$, the maximum principle yields $m(x) \leq \max \left(\frac{2 \max r}{k^{-}}, C\right)$ for $-a \leq x \leq 0$ and thus for $-a \leq x \leq a$. This uniform bound for the mass enables to argue exactly as in subsection 3.3 - recall that $c>c^{*}$ has been fixed - to get (59).

Uniform lower bound for $u_{b}(0,0)$. We choose $R>0$ large enough so that (42) holds, and $C>0$ such that (48) holds. Then, for $b \geq R+1, v_{b}(x, y):=u_{b}\left(\frac{x-B y}{\sqrt{B^{2}+1}}, \sqrt{B^{2}+1} y\right)$ satisfies (44) in the strip $S=\mathbb{R} \times\left(-\frac{R}{\sqrt{B^{2}+1}}, \frac{R}{\sqrt{B^{2}+1}}\right)$. We are therefore in the position to reproduce the proof of Proposition $4.3(i)$. Hence, there is $\kappa_{b}>0$, depending on $u_{b}(0,0)$, such that $v_{b}(x, y) \geq \kappa_{b} \Gamma_{R}^{0}(y)$ for all $(x, y) \in(-\infty, 0] \times[-R, R]$. Since $b \geq R+1$, we can apply the Harnack inequality to show that there exists $\varepsilon_{b}>0$ such that

$$
v_{b}(x, y) \geq \varepsilon_{b}, \quad \forall(x, y) \in(-\infty, 0] \times[-R, R] .
$$

Let us now introduce a smooth function $\phi: \mathbb{R} \rightarrow[0,1]$, such that $\phi \equiv 0$ on $(-\infty,-4] \cup[-1, \infty)$, and $\phi \equiv 1$ on $[-3,-2]$. For $\eta>0, \alpha \geq 0$, let us define

$$
\psi_{\alpha, \eta}(x, y):=\alpha \phi(\eta x) \Gamma_{R}^{0}\left(\sqrt{B^{2}+1} y\right) \quad \text { for }(x, y) \in \bar{S} .
$$

In view of (59) we have $v_{b} \leq M$ so that, for any $\eta>0$, we can define

$$
\alpha_{0}=\alpha_{0}(\eta, b):=\max \left\{\alpha \geq 0: \forall(x, y) \in \bar{S}, \psi_{\alpha, \eta}(x, y) \leq v_{b}(x, y)\right\} \in\left[0, \alpha_{\max }\right],
$$

with $\alpha_{\max }:=M /\left\|\Gamma_{R}^{0}\right\|_{\infty}$. Hence $v_{b}-\psi_{\alpha_{0}, \eta}$ attains a zero minimum at a point $\left(x_{0}, y_{0}\right)-$ depending on $\eta$ and $b$ - which must lie in $\left(-\frac{4}{\eta},-\frac{1}{\eta}\right) \times\left(-\frac{R}{\sqrt{B^{2}+1}}, \frac{R}{\sqrt{B^{2}+1}}\right)$, thanks to the definition of $\psi_{\alpha, \eta}$ and $\phi$. Then,

$$
0 \geq-\Delta_{x, y}\left(v_{b}-\psi_{\alpha_{0}, \eta}\right)\left(x_{0}, y_{0}\right)-c \sqrt{B^{2}+1} \partial_{x}\left(v_{b}-\psi_{\alpha_{0}, \eta}\right)\left(x_{0}, y_{0}\right) .
$$

Using (44), the first part of (42) and straightforward computations, we arrive at

$$
\begin{aligned}
0 \geq & \left(\frac{-\lambda_{\infty}^{0}}{4}-2 k^{+} C R v_{b}\left(x_{0}, y_{0}\right)\right) v_{b}\left(x_{0}, y_{0}\right)+ \\
& \alpha_{0}\left(\eta^{2} \phi^{\prime \prime}\left(\eta x_{0}\right)+\eta c \sqrt{B^{2}+1} \phi^{\prime}\left(\eta x_{0}\right)\right) \Gamma_{R}^{0}\left(\sqrt{B^{2}+1} y_{0}\right) .
\end{aligned}
$$

Then (60) yields

$$
0 \geq\left(\frac{-\lambda_{\infty}^{0}}{4}-2 k^{+} C R v_{b}\left(x_{0}, y_{0}\right)-\frac{M\left(\eta^{2}\left\|\phi^{\prime \prime}\right\|_{\infty}+\eta c \sqrt{B^{2}+1}\left\|\phi^{\prime}\right\|_{\infty}\right)}{\varepsilon_{b}}\right) v_{b}\left(x_{0}, y_{0}\right) .
$$


Since $\eta>0$ can be arbitrarily small, we discover that $v_{b}\left(x_{0}, y_{0}\right) \geq \frac{-\lambda^{0}}{8 k^{+} C R}$, which in turn implies $\alpha_{0} \geq \varepsilon:=\frac{-\lambda_{\infty}^{0}}{8 k^{+} C R\left\|\Gamma_{R}^{0}\right\|_{\infty}}$. Since $\psi_{\alpha_{0}, \eta}\left(-\frac{2}{\eta}, 0\right)=\alpha_{0}$ there is a point $x_{b}$ where $v_{b}\left(x_{b}, 0\right) \geq \varepsilon$, which in turn provides a point $x_{b}^{\prime}$ where $u_{b}\left(x_{b}^{\prime}, 0\right) \geq \varepsilon$. Since the problem in the strip is invariant w.r.t. translation in the $x$ variable, we can assume without loss of generality that $x_{b}^{\prime}=0$. Thus we have the desired lower uniform bound $u_{b}(0,0) \geq \varepsilon$.

Conclusion. The family $\left(u_{b}\right)_{b}$ is uniformly bounded, and is then uniformly bounded in $C^{2, \alpha}\left(\mathbb{R}^{2}\right)$, and we may pass to the limit $b \rightarrow \infty$, possibly along a subsequence. In this limit, we have $u_{b} \rightarrow u$, which is a solution of (49) in $\mathbb{R}^{2}$, and satisfies (58), (59) in $\mathbb{R}^{2}$ and $u(0,0) \geq \varepsilon>0$. Hence, we have constructed $u$ which satisfies (10), (13) - which in turn implies (12) - and the upper bound in (11). Last, the lower bound in (11) follows from Proposition $4.3(i)$. This concludes the proof of Theorem 1.4 in the case $c>c^{*}$.

Acknowledgements. M. A. is supported by the French Agence Nationale de la Recherche within the project IDEE (ANR-2010-0112-01). G. R. is partially supported by the French Agence Nationale de la Recherche within the project CBDif-Fr ANR-08-BLAN-0333-01. G. R. thanks Sepideh Mirrahimi for early discussions and computations on this problem.

\section{References}

[1] M. Alfaro and J. Coville, Rapid travelling waves in the nonlocal Fisher equation connect two unstable states, Appl. Math. Lett. 25 (2012), no. 12, 2095-2099.

[2] A. Arnold, L. Desvillettes and C. Prevost, Existence of nontrivial steady states for populations structured with respect to space and a continuous trait, Comm. Pure Appl. Anal. 11 (2012), no. 1, 83-96.

[3] D. G. Aronson and H. F. Weinberger, Multidimensional nonlinear diffusion arising in population genetics, Adv. in Math. 30 (1978), no. 1, 33-76.

[4] O. Benichou, V. Calvez, N. Meunier and R. Voituriez, Front acceleration by dynamic selection in Fisher population waves, Phys. Rev. E 86, 041908 (2012).

[5] H. Berestycki and G. Chapuisat, Traveling fronts guided by the environment for reactiondiffusion equations, submitted.

[6] H. Berestycki and F. Hamel, Front propagation in periodic excitable media, Comm. Pure Appl. Math. 55 (2002), no. 8, 949-1032.

[7] H. Berestycki and F. Hamel, Generalized transition waves and their properties, Comm. Pure Appl. Math. 65, (2012), no. 5, 592-648.

[8] H. Berestycki, F. Hamel and L. Roques, Analysis of the periodically fragmented environment model. II. Biological invasions and pulsating travelling fronts, J. Math. Pures Appl. (9) 84 (2005), no. 8, 1101-1146.

[9] H. Berestycki, G. Nadin, B. Perthame and L. Ryzhik, The non-local Fisher-KPP equation: travelling waves and steady states, Nonlinearity 22 (2009), no. 12, 2813-2844.

[10] H. Berestycki, B. Nicolaenko and B. Scheurer, Traveling wave solutions to combustion models and their singular limits, SIAM J. Math. Anal. 16 (1985), no. 6, 1207-1242.

[11] H. Berestycki and L. Nirenberg, On the method of moving planes and the sliding method, Bol. Soc. Brasil. Mat. (N.S.) 22 (1991), no. 1, 1-37.

[12] H. Berestycki and L. Nirenberg, Travelling fronts in cylinders, Ann. Inst. H. Poincaré Anal. Non Linéaire 9 (1992), no. 5, 497-572. 
[13] H. Berestycki, L. Nirenberg, and S. R. S. Varadhan, The principal eigenvalue and maximum principle for second-order elliptic operators in general domains, Comm. Pure Appl. Math. 47 (1994), no. 1, 47-92.

[14] H. Berestycki and L. Rossi, On the principal eigenvalue of elliptic operators in $\mathbb{R}^{N}$ and applications, J. Eur. Math. Soc. 8 (2006), no. 2, 195-215.

[15] E. Bouin, V. Calvez, N. Meunier, S. Mirrahimi, B. Perthame, G. Raoul and R. Voituriez, Invasion fronts with variable motility: phenotype selection, spatial sorting and wave acceleration, to appear in C. R. Math. Acad. Sci. Paris.

[16] N. Champagnat and S. Méléard, Invasion and adaptive evolution for individual-based spatially structured populations, J. Math. Biol. 55 (2007), 147-188.

[17] J. Coville, J. Dávila and S. Martínez, Nonlocal anisotropic dispersal with monostable nonlinearity, J. Differential Equations 244 (2008), no. 12, 3080-3118.

[18] J. Coville, J. Dávila and S. Martínez, Pulsating fronts for nonlocal dispersion and KPP nonlinearity, Annales de l'Institut Henri Poincare (C) Non Linear Analysis, 2012, doi:10.1016/j.anihpc.2012.07.005.

[19] J. Coville and L. Dupaigne, On a non-local reaction diffusion equation arising in population dynamics, Proc. Roy. Soc. Edinburgh Sect. A 137 (2007), no. 4, 727-755.

[20] M. B. Davis, R. G. Shaw and J. R. Etterson, Evolutionary responses to changing climate, Ecology 86 (2005), no. 7, 1704-1714.

[21] A. Duputié, F. Massol, I. Chuine, M. Kirkpatrick and O. Ronce, How do genetic correlations affect species range shifts in a changing environment?, Ecol. Lett. 15 (2012), $251-259$.

[22] J. R. Etterson, D. E. Delf, T. P. Craig, Y. Ando and T. Ohgushi, Parallel patterns of clinal variation in Solidago altissima in its native range in central U.S.A. and its invasive range in Japan, Botany $\mathbf{8 6}$ (2007), 91-97.

[23] R. A. Fisher, The wave of advance of advantageous genes, Ann. of Eugenics 7 (1937), 355-369.

[24] S. Genieys, V. Volpert and P. Auger, Pattern and waves for a model in population dynamics with nonlocal consumption of resources, Math. Model. Nat. Phenom. 1 (2006), no. $1,65-82$.

[25] D. Gilbarg and N. Trudinger, Elliptic Partial Differential Equations of Second Order, Springer-Verlag: Berlin, 1977.

[26] T. M. Griffith and M. A. Watson, Is evolution necessary for range expansion? Manipulating reproductive timing of a weedy annual transplanted beyond its range, Am. Nat. 167 (2006), no. 2, 153-164.

[27] R. Hermsen, J. B. Deris and T. Hwa, On the rapidity of antibiotic resistance evolution facilitated by a concentration gradient, Proc. Nat. Acad. Sci. USA 109 (2012), 1077510780 .

[28] W. Hudson and B. Zinner, Existence of traveling waves for reaction diffusion equations of Fisher type in periodic media, Boundary value problems for functional-differential equations, 187-199, World Sci. Publ., River Edge, NJ, 1995.

[29] P. E. Jabin and G. Raoul, On selection dynamics for competitive interactions, J. Math. Biol. 63 (2011), no. 3, 493-517. 
[30] S. R. Keller and D. R. Taylor, History, chance and adaptation during biological invasion: separating stochastic phenotypic evolution from response to selection, Ecol. lett. 11 (2008), 852-866.

[31] J. E. Keymer, P. Galajda, C. Muldoon, S. Park and R. H. Austin, Bacterial metapopulations in nanofabricated landscapes, Proc. Nat. Acad. Sci. USA 103 (2006), no. 46, 17290-17295.

[32] M. Kirkpatrick and N. H. Barton, Evolution of a species' range, Amer. Nat. 150 (1997), no. 1, 1-23.

[33] A. N. Kolmogorov, I. G. Petrovsky and N. S. Piskunov, Étude de l'équation de la diffusion avec croissance de la quantité de matière et son application à un problème biologique, Bulletin Université d'État à Moscow (Bjul. Moskowskogo Gos. Univ), Série Internationale, (1937), Section A, 1-26.

[34] A. Lorz, S. Mirrahimi and B. Perthame, Dirac mass dynamics in a multidimensional nonlocal parabolic equation, Comm. Partial Differential Equations 36 (2011), no. 6, 10711098.

[35] S. Mirrahimi and G. Raoul, Population structured by a space variable and a phenotypical trait, submitted.

[36] G. Nadin, Traveling fronts in space-time periodic media, J. Math. Pures Appl. (9) 92 (2009), no. 3, 232-262.

[37] J. Nolen and L. Ryzhik, Traveling waves in a one-dimensional heterogeneous medium, Ann. Inst. H. Poincaré Anal. Non Linéaire 26 (2009), no. 3, 1021-1047.

[38] J. R. Peck, J. M. Yearsley and D. Waxman, Explaining the geographic distributions of sexual and asexual populations, Nature 391 (1998), 889-892.

[39] B. L. Phillips, G. P. Brown, J. K. Webb and R. Shine, Invasion and the evolution of speed in toads, Nature 439 (803) (2006).

[40] J. Polechová and N. Barton, Speciation through competition: a critical review, Evolution 59 (2005), 1194-1210.

[41] C. Prevost, Applications of partial differential equations and their numerical simulations of population dynamics, PhD Thesis, University of Orleans (2004).

[42] W. Shen and A. Zhang, Traveling wave solutions of spatially periodic nonlocal monostable equations, ArXiv e-prints, (2012). http://arxiv.org/abs/1202.2452

[43] H.F. Weinberger, Long-time behavior of a class of biological models, SIAM J. Math. Anal. 13 (1982), no. 3, 353-396.

[44] J. Xin, Front propagation in heterogeneous media, SIAM Rev. 42, (2000), no. 2, 161-230. 